\title{
Random Van der Waerden Theorem
}

\author{
Ohad Zohar \\ Tel Aviv University, Israel \\ ohadvb@gmail.com
}

Submitted: Jul 27, 2020; Accepted: Nov 12, 2021; Published: Jan 28, 2022

(C) The author. Released under the CC BY-ND license (International 4.0).

\begin{abstract}
In this paper, we prove a sparse random analogue of the Van der Waerden Theorem. We show that, for all $r>2$ and all $q_{1} \geqslant q_{2} \geqslant \cdots \geqslant q_{r} \geqslant 3 \in \mathbb{N}, n^{-\frac{q_{2}}{q_{1}\left(q_{2}-1\right)}}$ is a threshold for the following property: For every $r$-coloring of the $p$-random subset of $\{1, \ldots, n\}$, there exists a monochromatic $q_{i}$-term arithmetic progression colored $i$, for some $i$. This extends the results of Rödl and Ruciński for the symmetric case $q_{1}=q_{2}=\cdots=q_{r}$. The proof of the 1-statement is based on the Hypergraph Container Method by Balogh, Morris and Samotij and Saxton and Thomason. The proof of the 0-statement is an extension of Rödl and Rucinski's argument for the symmetric case.
\end{abstract}

Mathematics Subject Classifications: 05C88, 05C89

\section{Introduction}

For $n, q \in \mathbb{N}$ and $r \geqslant 2$ let $[n] \rightarrow(q)_{r}$ denote the property that, for every $r$-coloring of $[n]=\{1, \ldots, n\}$, there exists a monochromatic arithmetic progression of length $q$. More generally, we denote by $[n] \rightarrow\left(q_{1}, \ldots, q_{r}\right)$ the property that, for every $r$-coloring of $[n]$, there exists $i$ such that there is some arithmetic progression of length $q_{i}$ colored $i$. A classical result in Ramsey theory due to Van der Waerden [22], states that, for every choice of $q_{1}, \ldots, q_{r} \in \mathbb{N}$, there exists $n_{0}$ such that for every $n \geqslant n_{0}$ we have $[n] \rightarrow\left(q_{1}, \ldots q_{r}\right)$. One might think of Van der Waerden's theorem as the arithmetic analogue to the graphtheoretic Ramsey Theorem.

This work aims to determine necessary and sufficient conditions for the property $[n] \rightarrow$ $\left(q_{1}, \ldots, q_{r}\right)$ to hold when we replace $[n]$ with a typical set of a given density. We define $A_{p}$ as the random subset of a set $A$, where every element $a \in A$ belongs to $A_{p}$ with probability $p$, independently of all other elements of $A$. Many interesting questions in Ramsey theory deal with determining the thresholds for values of $p$ for which it is no longer possible to color $A_{p}$ without introducing specific monochromatic substructures. 
Even though the use of probabilistic methods in Ramsey theory has a long history, the study of Ramsey properties of random structures was initiated more recently by Frankl and Rödl [5], who applied probabilistic methods to prove the existence of a graph $G$ with no $K_{4}$ for which every 2 -coloring must contain a monochromatic triangle.

In a series of papers, Rödl and Ruciński [17, 18, 19], determined the thresholds for the symmetric Van der Waerden property. Let $[n]_{p} \rightarrow(q)_{r}$ denote the event that, for every $r$-coloring of $[n]_{p}$, there exists a monochromatic arithmetic progression of length $q$. Similarly, let $[n]_{p} \rightarrow\left(q_{1}, \ldots, q_{r}\right)$ denote the event that, for every $r$-coloring of $[n]_{p}$, there exists a color $i$ such that there exists an arithmetic progression of length $q_{i}$ colored $i$.

Theorem 1 (Rödl and Ruciński). For $3 \leqslant q \in \mathbb{N}$ and every $r \geqslant 2$, there exist $c, C>0$ such that

$$
\lim _{n \rightarrow \infty} \mathbb{P}\left([n]_{p} \rightarrow(q)_{r}\right)= \begin{cases}1 & \text { if } p \geqslant C \cdot n^{-\frac{1}{q-1}} \\ 0 & \text { if } p \leqslant c \cdot n^{-\frac{1}{q-1}}\end{cases}
$$

Similarly to the notation for the Van der Waerden property, one lets $G \rightarrow\left(F_{1}, \ldots, F_{r}\right)$ denote the property that, for every $r$-coloring of $E(G)$, there exists $i$ such that there is a copy of $F_{i}$ colored $i$. As well as Theorem 1, Rödl and Ruciński have also determined the threshold for the event $G(n, p) \rightarrow(F, \ldots, F)$. In 1997, Kohayakawa and Kreuter [11] initiated the study of the asymmetric case for graphs; they determined the threshold for the event $G(n, p) \rightarrow\left(C_{1}, \ldots, C_{r}\right)$ where $C_{1}, \ldots, C_{r}$ are all cycles, and conjectured the location of the threshold for general subgraphs $\left(F_{1}, \ldots, F_{r}\right)$. Several papers have since extended Kohayakawa and Kreuter's result to other families of subgraphs. For instance, Marciniszyn, Skokan, Spöhel and Steger [13], showed that the conjecture holds when $F_{1}, \ldots, F_{r}$ are all cliques. More recently Mousset, Nenadov and Samotij [14] proved an upper bound for the threshold function in the Kohayakawa-Kreuter conjecture for general subgraphs, extending a result of Gugelmann, Nenadov, Person, Škorić, Steger and Thomas [7], and settling the 1-statement. Very recently, Liebenau, Mattos, Mendonça and Skokan [12], have shown that the 0-statement holds for $r=2$ for any pair of cycles and cliques. However, the 0-statement for general subgraphs remains open.

In this paper we prove the following natural analogue of the Kohayakawa-Kreuter conjecture for the Van der Waerden theorem:

Theorem 2. For every $r \geqslant 2$ and $q_{1} \geqslant q_{2} \geqslant \cdots \geqslant q_{r} \in \mathbb{N}$, there exist $c, C>0$ such that

$$
\lim _{n \rightarrow \infty} \mathbb{P}\left([n]_{p} \rightarrow\left(q_{1}, \ldots, q_{r}\right)\right)= \begin{cases}1 & \text { if } p \geqslant C \cdot n^{-\frac{q_{2}}{q_{1}\left(q_{2}-1\right)}}, \\ 0 & \text { if } p \leqslant c \cdot n^{-\frac{q_{2}}{q_{1}\left(q_{2}-1\right)}} .\end{cases}
$$

Whether the threshold in Theorem 2 is sharp remains an interesting open question; so far it has only been shown that this is the case in $\mathbb{Z}_{n}$ when $r=2$ and $q_{1}=q_{2}$ by Friedgut, Hàn, Person and Schacht [6].

It is important to note that only the two largest lengths determine the threshold. Therefore, in the proof of the 1-statement, it suffices to assume $q_{2}=\cdots=q_{r}$. For the 0 -statement, we will show that a proper coloring exists using only the first two colors, as 
is necessary for the case $r=2$, since that is also sufficient for all values of $r$. By that reasoning, it is natural to divide the proof into the symmetric $\left(q_{1}=q_{2}\right)$ and asymmetric $\left(q_{1}>q_{2}\right)$ cases.

A generalization of Van der Waerden's theorem is the classical Rado theorem [16], which characterizes the so-called partition-regular matrices. A matrix $A$ is partitionregular if every finite coloring of the positive integers admits a monochromatic solution to the equation $A x=0$. Independently to this work, some progress has been made towards an asymmetric version of the random Rado theorem: given a sequence of partition-regular matrices $A_{1}, \ldots, A_{r}$, for which values of $p$ does every $r$-coloring of $[n]_{p}$ admit a solution to $A_{i} x=0$ colored $i$ ? Aigner-Horev and Person [1] obtained an upper bound for the threshold value, which implies the 1-statement in Theorem 2. Soon after, Hancock and Treglown [8] obtained a matching lower bound for the case where every $A_{i}$ has rank one; this result implies the 0 -statement in a special case of theorems 1 and 2 , where $q_{i}=3$ for all $i$.

The structure of this paper is as follows. In Section 2, we present several known results that are used in our proofs. In Sections 3 and 4, we present short proofs for the symmetric case, already proved by Rödl and Ruciński [18]. We also correct an error in their proof for the 0 -statement, which was independently discovered by Hancock and Treglown. These sections are included here strictly for completeness; readers familiar with these results are encouraged to continue reading from Section 5 in which we prove the 1-statement for the asymmetric case. Finally, in Section 6, we complete the proof of Theorem 2 by proving the 0 -statement for the asymmetric case.

\section{Preliminary results}

In this section, we state several known results that are used throughout this paper. The first result is the Hypergraph Container Lemma proved by Balogh, Morris and Samotij [3], and independently by Saxton and Thomason [21]. For an introduction to the various applications of this lemma, as well as the formulation used in this paper, we refer the reader to [4].

Definition 3. For a $k$-uniform hypergraph $H$ and a set $A \subset V(H)$ we define

$$
d(A)=|\{e \in E(H): A \subset e\}|,
$$

and for $\ell \in\{1, \ldots, k\}$ we define

$$
\Delta_{\ell}(H)=\max \{d(A): A \subset V(H) \text { and }|A|=\ell\} .
$$

Definition 4. Let $H$ be a hypergraph, we denote the set of independent subsets of $V(H)$ by

$$
\mathcal{I}(H)=\{I \subset V(H): \forall E \in E(H), E \not \subset I\} .
$$

Theorem 5 (The Hypergraph Container Lemma). Let $k \in \mathbb{N}$ and $\epsilon>0$. Let $H$ be $a$ nonempty $k$-uniform hypergraph, and suppose that: 


$$
\Delta_{\ell}(H) \leqslant K \cdot\left(\frac{b}{v(H)}\right)^{\ell-1} \cdot \frac{e(H)}{v(H)}
$$

for some $b, K \in \mathbb{N}$ and every $\ell \in\{1, \ldots, k\}$. Then, there exists a constant $D=D(\epsilon, k, K)$, a collection $\mathcal{C} \subset P(V(H))$ and a function $f: P(V(H)) \rightarrow \mathcal{C}$ such that:

1. for every $I \in \mathcal{I}(H)$, there exists $S \subset I$ with $|S| \leqslant D b$ and $I \subset f(S)$,

2. each $C \in \mathcal{C}$ contains fewer than $\epsilon \cdot e(H)$ edges.

Claim 6. Let $H$ be the hypergraph encoding $q$-APs in $[n]$, where $V(H)=[n]$ and $E(H)$ is the set of arithmetic progressions of length $q$. Then,

$$
\Delta(H)=\Delta_{1}(H) \leqslant n
$$

Proof. We denote the number of arithmetic progressions of length $q$ in $[n]$ such that $k$ is the $i$ th element by $d(k, i)$. One easily checks that $d(k, i)$ satisfies:

$$
d(k, i)= \begin{cases}\left\lfloor\frac{n-k}{q-1}\right\rfloor & \text { if } i=1, \\ \left\lfloor\frac{k-1}{q-1}\right\rfloor & \text { if } i=q, \\ \min \left\{\left\lfloor\frac{k-1}{i-1}\right\rfloor,\left\lfloor\frac{n-k}{q-i}\right\rfloor\right\} & \text { otherwise. }\end{cases}
$$

We obtain that that the degree of $k$ in $H$ satisfies

$$
d(k)=\sum_{i=1}^{q} d(k, i) \leqslant \frac{n}{q-1}+\sum_{i=2}^{q-1} d(k, i) .
$$

Applying the bound $\min \left\{\left\lfloor\frac{k-1}{i-1}\right\rfloor,\left\lfloor\frac{n-k}{q-i}\right\rfloor\right\} \leqslant \frac{k-1+n-k}{i-1+q-i}<\frac{n}{q-1}$ we conclude that

$$
d(k)=\sum_{i=1}^{q} d(k, i) \leqslant \frac{n}{q-1}+\sum_{i=2}^{q-1} d(k, i) \leqslant \frac{n}{q-1}+(q-2) \cdot \frac{n}{q-1} \leqslant n .
$$

Remark 7 . Let $H$ be the hypergraph encoding $q$-APs in $[n]$, where $V(H)=[n]$ and $E(H)$ is the set of arithmetic progressions of length $q$. Then:

1. The number of edges of $H$ satisfies

$$
e(H)=\sum_{i=1}^{n-q+1}\left\lfloor\frac{n-i}{q-1}\right\rfloor=\Theta\left(n^{2}\right)
$$

since there are $\left\lfloor\frac{n-a}{q-1}\right\rfloor$ arithmetic progressions of length $q$ in $[n]$ with smallest element $a$. 
2. For $\ell=1$, we have $\Delta_{1}(H) \leqslant n$, by the previous claim.

3. For every $\ell \geqslant 2$, we have $\Delta_{\ell}(H) \leqslant q^{2}$ since choosing the indices of two elements determines the arithmetic progression.

Therefore we may apply the Hypergraph Container Lemma with $b=q n^{\frac{q-2}{q-1}}$, since for $\ell=1$

$$
\Delta_{1}(H) \leqslant n \leqslant K \cdot \frac{e(H)}{v(H)}
$$

and for $2 \leqslant \ell \leqslant q$

$$
\Delta_{\ell}(H) \leqslant q^{2} \leqslant q^{q-1} \leqslant K \cdot \frac{q^{q-1} n^{q-2}}{n^{q-1}} \cdot \frac{e(H)}{n} \leqslant K \cdot\left(\frac{q n^{q-2}}{v(H)}\right)^{\ell-1} \cdot \frac{e(H)}{v(H)},
$$

provided that $K$ and $n$ are sufficiently large.

Definition 8. For brevity, we say a set of integers is $q$-AP-free if it contains no arithmetic progression of length $q$.

We obtain the following container lemma for arithmetic progressions.

Theorem 9. For every integer $q \geqslant 3$ and $\epsilon>0$, there exists a constant $D=D(\epsilon, q)$ such that for each $n \in \mathbb{N}$, there exists a collection $\mathcal{G} \subset P([n])$ and a function $f: P([n]) \rightarrow \mathcal{G}$ such that:

1. each $G \in \mathcal{G}$ contains fewer then $\epsilon n^{2}$ many $q$-APs,

2. for every $q$-AP-free subset $I \subset[n]$, there exists $S \subset I$ with $|S| \leqslant D \cdot n^{\frac{q-2}{q-1}}$ and $I \subset f(S)$.

We also require two classical results in probabilistic combinatorics, the first of which is Janson's inequality [10].

Theorem 10 (Janson's inequality). Let $\Gamma$ be a finite set and let $\mathcal{S} \subset P(\Gamma)$. For every $A \in \mathcal{S}$, let $I_{A}=1$ if $A \subset \Gamma_{p}$ and $I_{A}=0$ otherwise.

Let $X=\sum_{A \in \mathcal{S}} I_{A}$ be the random variable counting the sets of $\mathcal{S}$ which are entirely contained in $\Gamma_{p}$. Set

$$
\mu=\mathbb{E} X \quad \text { and } \quad \Delta=\sum_{\substack{(A, B) \in \mathcal{S}^{2} \\ A \neq B, A \cap B \neq \emptyset}} \mathbb{E}\left[I_{A} \cdot I_{B}\right]
$$

Then,

$$
\mathbb{P}(X=0) \leqslant e^{-\mu+\frac{\Delta}{2}} .
$$

Moreover, if $\Delta \geqslant \mu$, then

$$
\mathbb{P}(X=0) \leqslant e^{-\frac{\mu^{2}}{2 \Delta}}
$$


The second inequality is also known as The Extended Janson Inequality. For an introduction, as well as proofs for both inequalities, we refer the reader to [2].

We will also require a special case of Harris's inequality [9].

Theorem 11 (Harris's inequality). Let $\Gamma$ be a finite set and let $f: P(\Gamma) \rightarrow\{0,1\}$ be an indicator function for some family of sets $\mathcal{A}$. We say $\mathcal{A}$ is increasing (equivalently decreasing), if $S_{1} \subset S_{2} \Longrightarrow f\left(S_{1}\right) \leqslant f\left(S_{2}\right)$ (equivalently $f\left(S_{1}\right) \geqslant f\left(S_{2}\right)$ ). If $\mathcal{A}$ is increasing and $\mathcal{B}$ is decreasing, then

$$
\mathbb{P}\left(\Gamma_{p} \in \mathcal{A} \cap \mathcal{B}\right) \leqslant \mathbb{P}\left(\Gamma_{p} \in \mathcal{A}\right) \cdot \mathbb{P}\left(\Gamma_{p} \in \mathcal{B}\right) .
$$

Again, for a more detailed discussion, we refer the reader to [2].

The final result in this section is a well-known quantitative version of Van der Waerden's Theorem due to Varnavides [23].

Lemma 12. For every $r \in \mathbb{N}$ and every $q \geqslant 2$, there exist $n_{0} \in \mathbb{N}$ and $\epsilon>0$ such that for all $n \geqslant n_{0}$, every $(r+1)$-coloring of $[n]$ contains at least $(r+1) \cdot \epsilon \cdot n^{2}$ monochromatic arithmetic progressions of length $q$.

Proof. From Van der Waerden's theorem we have $W=W(r+1, q)$ such that every $(r+1)$ coloring of $[W]$ yields a monochromatic $q$-AP. Thus, in every coloring of $[n]$ every $W$-AP contains at least one monochromatic $q$-AP. We observe:

(a) $\# W$-APs in $[n]=\Theta\left(\frac{n(n-W)}{W-1}\right)$.

(b) Every $q$-AP is contained in at most $W^{2}$ many $W$-APs (fixing the indices of two terms in an arithmetic progression determines the progression).

We obtain from (a) $\Theta\left(\frac{1}{W} \cdot n^{2}\right)$ many $q$-APs, however they may be contained in multiple $W$-APs. Since by (b) every $q$-AP is counted at most $W^{2}$ times, the lemma follows with $\epsilon=\Theta\left(\frac{W^{-3}}{2(r+1)}\right)$.

\section{The symmetric case 1 -statement}

In this section, we present a short proof to the following theorem (originally proved by Rödl and Ruciński [19]):

Theorem 13. For every $r \in \mathbb{N}$ and $q \geqslant 3$, there exists $C>0$ such that the following holds: If $p \geqslant C n^{-\frac{1}{q-1}}$ then a.a.s. every r-coloring of $[n]_{p}$ contains a monochromatic $q-A P$.

The general framework of the proof we present here is due to Nenadov and Steger [15] who applied a similar argument in the setting of graphs. We begin by describing a general outline of the proof.

From The Hypergraph Container Lemma, we obtain a set of containers $\mathcal{G}$ for $q$-AP-free subsets of $[n]$. Assume for contradiction that $[n]_{p}$ has a coloring with no monochromatic 
arithmetic progression of length $q$, and fix an arbitrary such coloring. Let $G_{i} \in \mathcal{G}$ be the container for the $i$-th color class. Each container contains strictly fewer than $\epsilon n^{2}$ many $q$-term arithmetic progressions. However, by Lemma 12 every coloring in $r+1$ colors must contain some color class with at least $\epsilon n^{2}$ arithmetic progressions. In our case, we treat the remainder set $[n] \backslash \bigcup_{i} G_{i}$ as the final color class. Hence, it must have at least $\epsilon n^{2}$ arithmetic progressions and therefore has at least $\epsilon n$ many elements. However, by definition, the remainder set (which depends only on $G_{1}, \ldots, G_{r}$ ) and $[n]_{p}$ are disjoint. Thus, the probability that $[n]_{p}$ has a coloring with no monochromatic $q$-term arithmetic progression that obeys the coloring constraints set by $\left(G_{1}, \ldots, G_{r}\right)$ is at most $(1-p)^{\epsilon n}$. Finally, we apply a union bound over all possible choices for the $r$-tuple of containers, obtaining that the probability that such $[n]_{p}$ has a proper $r$-coloring tends to zero.

Proof of Theorem 13. We say that a coloring is "proper" if it contains no monochromatic $q$-AP. We wish to prove that a.a.s. $[n]_{p}$ admits no such coloring. Applying Theorem 9 with $\epsilon=\epsilon(r)$ obtained from Lemma 12, we obtain a family of containers $\mathcal{G}$ such that each $G \in \mathcal{G}$ contains fewer than $\epsilon n^{2}$ many $q$-APs. If we suppose that there exists a proper coloring for $[n]_{p}$, then there are $q$-AP-free subsets $H_{1}, \ldots, H_{r}$, such that $\bigcup_{i=1}^{r} H_{i}=[n]_{p}$.

By Theorem 9, there exist a constant $D$ and a function $f: P([n]) \rightarrow \mathcal{G}$ such that for every $i$ there exists a set $S_{i} \subset H_{i}$, with $\left|S_{i}\right| \leqslant D \cdot n^{\frac{q-2}{q-1}}$ and $H_{i} \subset f\left(S_{i}\right)=G_{i}$. By Lemma 12 , we obtain that for any coloring of $[n]$ with $r+1$ colors there must be a color class with at least $\epsilon n^{2}$ arithmetic progressions.

Suppose we color the elements of $\bigcup G_{i}$ such that only elements of $G_{i}$ are colored $i$ and the elements of $[n] \backslash \bigcup_{i=1}^{r} G_{i}$ are colored $r+1$. Since every $G_{i}$ contains fewer than $\epsilon n^{2}$ arithemetic progressions, the set of elements colored $r+1$ must contain $\epsilon n^{2}$ arithmetic progressions. Since, by Claim 6 , each $k \in[n]$ belongs to at most $n$ many $q$-APs we have

$$
\left|[n] \backslash \bigcup_{i=1}^{r} G_{i}\right| \geqslant \frac{\epsilon n^{2}}{n}=\epsilon n .
$$

In summary, the event "There exists a proper coloring of $[n]_{p}$ " implies the following two events, for some $S_{1}, \ldots, S_{r} \subset[n]$ with $\left|S_{i}\right| \leqslant D n^{\frac{q-2}{q-1}}$ for every $i \in\{1, \ldots, r\}$ :

1. $\bigcup_{i=1}^{r} S_{i} \subset[n]_{p}$,

2. $[n]_{p} \subset \bigcup_{i=1}^{r} f\left(S_{i}\right)$.

Note that (b) is equivalent to

$$
[n]_{p} \cap\left([n] \backslash \bigcup_{i=1}^{r} f\left(S_{i}\right)\right)=\emptyset .
$$

Since $S_{i} \subset f\left(S_{i}\right)$ for every $i,(\mathrm{a})$ and (b) depend on disjoint subsets of $[n]$ and are independent events. Hence, the probability of both (a) and (b) occurring is: 


$$
\mathbb{P}\left(S_{1}, \ldots, S_{r} \subset[n]_{p} \wedge[n]_{p} \subset \bigcup_{i=1}^{r} f\left(S_{i}\right)\right) \leqslant p^{\left|\bigcup_{i=1}^{r} S_{i}\right|} \cdot(1-p)^{\epsilon n} .
$$

Givan a set $S$ with $|S|=s$, there are at most $2^{r s}$ sequences $\left(S_{1}, \ldots, S_{r}\right)$ such that $S=\bigcup S_{i}$. Taking the union bound over all choices of $S_{1}, \ldots, S_{r}$ (grouping by $\left.s=\left|\bigcup_{i=1}^{r} S_{i}\right|\right)$ we obtain

$$
\begin{gathered}
\mathbb{P}\left([n]_{p} \text { admits a proper coloring }\right) \leqslant \sum_{\left(S_{1}, \ldots, S_{r}\right)} p^{\left|\bigcup_{i=1}^{r} S_{i}\right|} \cdot(1-p)^{\epsilon n} \\
\leqslant(1-p)^{\epsilon n} \sum_{s=1}^{D r n^{\frac{q-2}{q-1}}}\left(\begin{array}{c}
n \\
s
\end{array}\right) 2^{r s} p^{s} \leqslant e^{-\epsilon p n} \sum_{s=1}^{\operatorname{Drn} \frac{q-2}{q-1}}\left(\frac{e n 2^{r} p}{s}\right)^{s} .
\end{gathered}
$$

Recall that $p=C n^{-\frac{1}{q-1}}$. Since $x \mapsto\left(\frac{e a}{x}\right)^{x}$ is increasing for $x \leqslant a$, by choosing $C$ sufficiently large we obtain that $D r n^{\frac{q-2}{q-1}} \leqslant \delta p n$ for some $\delta=\delta(C, D, r)>0$ which can be made arbitrarily small by choosing $C$ sufficiently large. Hence,

$$
\begin{aligned}
\sum_{s=1}^{D r n} \frac{q-2}{q-1}\left(\frac{e n 2^{r} p}{s}\right)^{s} & \leqslant D r n^{\frac{q-2}{q-1}} \cdot\left(\frac{e n 2^{r} p}{D r n^{\frac{q-2}{q-1}}}\right)^{\operatorname{Drn} n^{\frac{q-2}{q-1}}} \\
& \leqslant \delta p n \cdot\left(\frac{e 2^{r}}{\delta}\right)^{\delta p n} \leqslant e^{\frac{\epsilon p n}{2}}
\end{aligned}
$$

for sufficiently large $C$, since $\left(\frac{1}{\delta}\right)^{\delta} \rightarrow 1$ as $\delta \rightarrow 0$. Hence:

$\mathbb{P}\left([n]_{p}\right.$ admits a proper coloring $) \leqslant e^{\frac{-\epsilon p n}{2}} \rightarrow 0$.

\section{The symmetric case 0 -statement}

In this section, we prove the following theorem (originally proved by Rödl and Ruciński [19]):

Theorem 14. For any integer $q \geqslant 3$, there exists $c>0$ such that for $p=c \cdot n^{-\frac{1}{q-1}},[n]_{p}$ can a.a.s. be colored by two colors with no monochromatic q-term arithmetic progression.

Note that this implies the 0 -statement in Theorem 1 for any $r \geqslant 2$. The proof here is a specialization of the proof by Rödl and Ruciński [20] to the random Rado partition theorem, and is included here both for the sake of completeness and as an introduction to the techniques used for the asymmetric case in Section 6. The proof consists of two main lemmas. The deterministic lemma states that every non-2-colorable uniform hypergraph must contain one of a small family of hypergraphs which we refer to as 2-blocking hypergraphs (which will be defined next). Then, the probabilistic lemma states that in the random hypergraph of arithmetic progressions in $[n]_{p}$, the subhypergraphs mentioned in the deterministic lemma almost surely do not appear. We begin by defining several families of hypergraphs. 
Definition 15. A simple path is a hypergraph consisting of edges $E_{1}, \ldots, E_{\ell}$, for $\ell \geqslant 1$, such that

$$
\left|E_{i} \cap E_{j}\right|= \begin{cases}1 & \text { if }|i-j|=1, \\ 0 & \text { if otherwise. }\end{cases}
$$

A fairly simple cycle is a hypergraph that consists of a simple path $\left(E_{1}, \ldots, E_{\ell}\right)$, with $\ell \geqslant 2$, and an edge $E_{0}$ such that

$$
\left|E_{0} \cap E_{i}\right|= \begin{cases}1 & \text { if } i=1 \\ 0 & \text { if } i=2, \ldots, \ell-1, \\ s & \text { if } i=\ell\end{cases}
$$

for some $s \geqslant 1$, and such that $E_{0} \cap E_{1} \cap E_{\ell}=\emptyset$. A fairly simple cycle is said to be simple if $s=1$; otherwise, we say it is special. A path $P$ in a hypergraph $H$ is said to be spoiled if it is not an induced subhypergraph of $H$. We call an edge $E \subset V(P)$ such that $E \notin E(P)$ a spoiling edge for $P$.

The length of a path or a fairly simple cycle is the number of edges in it. A subhypergraph $H_{0}$ of $H$ is said to have a handle if there is an edge $E$ in $\mathrm{H}$ such that $|E|>\left|E \cap V\left(H_{0}\right)\right| \geqslant 2$.

Definition 16. We call a $q$-uniform hypergraph 2-blocking if it is one of the following:

1. A special cycle.

2. A simple cycle with a handle.

3. A spoiled path.

4. For $q=3$, the 3-uniform, 2-regular, 6-vertex, simple hypergraph, which we call "the reduced Fano plane" (See Figure 1a) ${ }^{1}$.

Lemma 17 (The determinisic lemma). Let $q \geqslant 3$ be some integer, and let $H$ be a $q$ uniform hypergraph which is not 2-colorable. Then $H$ contains a 2-blocking hypergraph.

Proof. Recall that we say a hypergraph is 3-edge-critical if it cannot be properly colored with two colors, but any proper subhypergraph of it is 2-colorable. We may assume that $H$ is 3 -edge-critical; otherwise, we may replace it with one of its 3 -edge-critical subhypergraphs.

Claim 18. If $H$ is a 3-edge-critical hypergraph, then for every edge $E \in H$ and for every vertex $v \in E$ there is an edge $E^{\prime} \in H$ such that $E \cap E^{\prime}=\{v\}$.

\footnotetext{
${ }^{1}$ The name stems from the fact that this hypergraph is exactly the Fano plane with one vertex removed. It is also sometimes refered to as a Pasch configuration.
} 
Proof. Let $H$ be 3-edge-critical, and suppose that there are an edge $E \in H$ and a vertex $v \in E$ such that no other edge intersects $E$ in exactly $\{v\}$; thus, every edge $E^{\prime}$ that contains $v$ also contains another vertex of $E$. By the 3 -edge-criticality, $H$ can be colored red-blue in a way such that only $E$ is monochoromatic, say it is blue. Now, by changing the color of $v$ to red, $E$ is no longer monochromatic, and neither is any other edge that contains $v$, contradicting the fact that $H$ is not 2-colorable.

Let $P=\left(E_{1}, \ldots, E_{\ell}\right)$ be a longest simple path in $H$. By the claim, $\ell \geqslant 2$. Let $x, y$ be two of the vertices that belong only to $E_{1}$, and let $E_{x}, E_{y}$ be two edges of $H$ that intersect $E_{1}$ only in $x$ and $y$ respectively. Since $P$ is maximal, $h_{z}=\left|E_{z} \cap V(P)\right| \geqslant 2$, for $z=x, y$.

We may also assume that $E_{x}, E_{y}$ only intersect one another and each edge of $P$ in at most a single vertex (otherwise we obtain a special cycle). Let $i_{z}=\min \{i \geqslant 2$ : $E_{z} \cap E_{i} \neq \emptyset$, and without loss of generality assume $i_{y} \leqslant i_{x}$. If $h_{z}=q$ for some $z$ then $P$ is a spoiled simple path. Otherwise, either the edges $E_{1}, \ldots, E_{i_{y}}, \ldots, E_{i_{x}}, E_{x}$ form a fairly simple cycle, to which $E_{y}$ is a handle (see Figure 1b) or $E_{y} \subset\left(E_{1} \cup \cdots \cup E_{i_{x}} \cup E_{x}\right)$.

Assuming the latter case, since $h_{y}<q$ and we assumed that $\left|E_{x} \cap E_{y}\right| \leqslant 1$, we must have $E_{x} \cap E_{y}=\{u\}$ for some vertex $u \notin V(P)$. We now split into several cases. First, if $i_{y}<i_{x}$ then the edges $E_{1}, \ldots, E_{i_{y}}, E_{y}$ form a fairly simple cycle to which $E_{x}$ is a handle (since it only intersects the cycle at $x$ and $u$ ). Finally, if $i_{y}=i_{x}=i$ then either $E_{x}, E_{y}, E_{i}$ form a fairly simple cycle to which $E_{1}$ is a handle, or $i=2, q=3$ and $E_{1}, E_{2}, E_{x}, E_{y}$ form the reduced Fano plane.

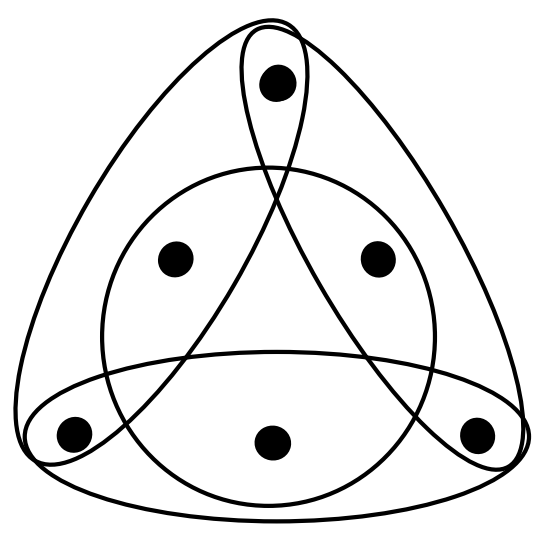

(a) The reduced Fano plane

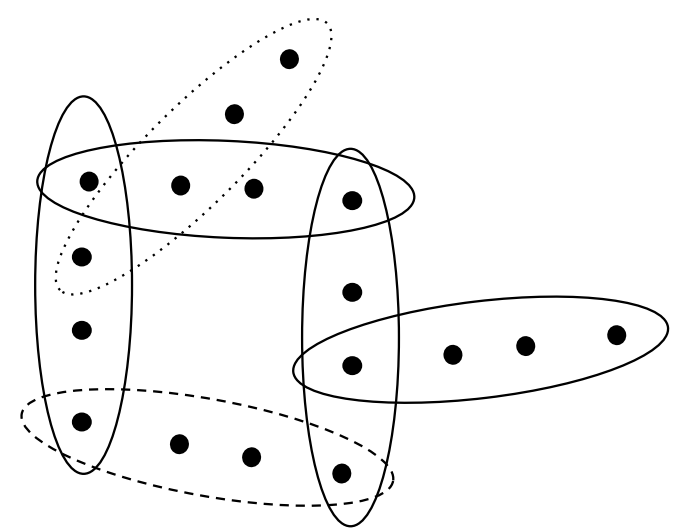

(b) $E_{x}$ (dashed) forms a cycle with $P$ to which $E_{y}$ (dotted) is a handle.

Lemma 19 (The probabilistic lemma). Let $H$ be the hypergraph with vertex set $V(H)=$ $[n]$, whose edge set is the set of $q-A P s$, and let $H_{p}$ be its random subhypergraph induced by $[n]_{p}$. If $p=c \cdot n^{-\frac{1}{q-1}}$, then a.a.s. $H_{p}$ contains no 2-blocking hypergraph, provided that $c$ is sufficiently small. 
Proof. We apply a first-moment argument to several random variables. Let $B$ be a large enough constant, we will show that a.a.s. no path of length $B \log n$ exists in $H_{p}$. We will then show that a.a.s. no 2-blocking graph with fewer than $B \log n$ edges exist.

The following calculations are used several times throughout the arguments. First, the number of choices for a $q$-term arithmetic progression in $[n]$ is $O\left(n^{2}\right)$, and the probability that all of its $q$ elements belong to $[n]_{p}$ is $\left(c n^{-\frac{1}{q-1}}\right)^{q}=O\left(n^{-1} p\right)$, so the expected number of $q$-term arithmetic progressions in $[n]_{p}$ is $O(n p)$.

We say that an edge $A$ extends a simple path $P=\left(E_{1}, \ldots, E_{\ell}\right)$ in $H$, if $A \cap V(P)=\{v\}$ and $\left\{i: v \in E_{i}\right\}$ is exactly $\{1\}$ or $\{\ell\}$. Observe that the number of choices for an arithmetic progression $A$ that extends a simple path $P$ is bounded by $2 \cdot(q-1) \cdot q \cdot n$, and that the probability that $A \backslash V(P) \subset[n]_{p}$ is $\left(c n^{-\frac{1}{q-1}}\right)^{q-1}$. Therefore, we may bound the expected number of possible edge choices for extending a simple path in $H_{p}$ by some constant $c_{r}$, which may depend on $q$ but not on $n$, and may be made arbitrarily small by changing $c$.

The number of arithmetic progressions containing a fixed set $\left\{v_{1}, \ldots, v_{s}\right\}$ with $s \geqslant$ 2 , is bounded by a constant (for instance $\left(\begin{array}{l}q \\ s\end{array}\right)$ is a trivial bound). Hence the expected number of arithmetic progressions in $H_{p}$ that contain $\left\{v_{1}, \ldots, v_{s}\right\}$ is $O\left(p^{q-s}\right)$, assuming $\left\{v_{1}, \ldots, v_{s}\right\} \subset[n]_{p}$.

Since the number of simple paths of length $t$ in $H$ is at most $O\left(n^{1+t}\right)$, and a simple path of length $t$ must contain $q$ vertices for the first edge and $q-1$ vertices for every subsequent edge, the expected number of simple paths of length $t$ in $H_{p}$ is

$$
O\left(n^{1+t} \cdot p^{q+(t-1)(q-1)}\right)=O\left(n p \cdot c_{r}^{t}\right) .
$$

We are now ready to proceed with the argument.

Let $U$ be the random variable counting the number of simple paths in $H_{p}$ of length at least $B \cdot \log n$. We bound the expected value of $U$ by summing over different lengths of paths. By the above computations,

$$
\mathbb{E} U \leqslant O\left(\sum_{t \geqslant B \log n} n p \cdot c_{r}^{t}\right)=o(1)
$$

provided that $c_{r}$ is sufficiently small and $B$ is sufficiently large.

Let $W$ be the random variable counting the number of special cycles in $H_{p}$. For a given edge there are only a constant number of edges that interesect it in more than one vertex, therefore the number of such edge pairs in $H$ is $O\left(n^{2}\right)$. We may bound the number of special cycles of length $t$ in $H$ by first fixing $E_{0}$ and $E_{t-1}$, then fixing a simple path of length $t-3$ starting from some vertex of $E_{0}$ and finally choosing $E_{t-2}$. Note that we have $O(1)$ many choices for $E_{t-2}$ since it must include exactly one vertex from $E_{t-3}$ and one vertex from $E_{t-1}$. Moreover, these vertices cannot coincide as $E_{0} \cap E_{1} \cap E_{t-1}=\emptyset$. In total we obtain that there are $O\left(n^{2} \cdot n^{t-3}\right)=O\left(n^{t-1}\right)$ such cycles in $H$. Since we must have at least $q+1$ vertices for $E_{0}$ and $E_{t-1}$, an additional $(t-3) \cdot(q-1)$ vertices for $E_{1}, \cdots, E_{t-3}$, and exactly $q-2$ additional vertices for $E_{t-2}$ we require a total of $2 q-1+(t-3) \cdot(q-1)=1+(t-1) \cdot(q-1)$ vertices in $H_{p}$. Hence,

$$
\mathbb{E} W=O\left(\sum_{t>2} n^{t-1} p^{1+(t-1) \cdot(q-1)}\right)=O\left(p \sum_{t>2} c_{r}^{t-1}\right)=O(p)=o(1)
$$


provided that $c_{r}$ is sufficiently small.

Let $X$ be the random variable counting the number of simple cycles with handles of length at most $B \log n$ in $H_{p}$. We denote the length of the cycle by $t$, and the size of the intersection between the handle and the cycle by $k$. Similarly to the previous argument, there are $O\left(n^{t}\right)$ many cycles of length $t$ in $H$. Since the handle must attach in at least two vertices of the cycle we may bound the number of handles in $H$ by $O\left(\log ^{2} n\right)$. For fixed values of $t$ and $k$, such a configuration requires $t \cdot(q-1)$ vertices for the cycle and an additional $q-k$ vertices for the handle. Summing over $t$ and $k$,

$$
\mathbb{E} X=O\left(\sum_{t=3}^{B \log n} \sum_{2 \leqslant k \leqslant q-1} n^{t} \cdot p^{t \cdot(q-1)+q-k} \cdot \log ^{2} n\right)=O\left(p \log ^{3} n\right)=o(1) .
$$

Let $Y$ be the random variable counting the number of spoiled simple paths of length less than $B \log n$ in $H_{p}$. Let $E$ be an induced edge that is not one of the edges of a spoiled path. We now split into two cases. First, suppose that $E$ intersects some edge in at least two vertices, we obtain a path $\left(E_{1}, \ldots, E_{\ell}\right)$ and an edge $E_{0}=E$, such that $\left|E_{0} \cap E_{1}\right|=s \geqslant 2,\left|E_{0} \cap\left(E_{\ell} \backslash E_{1}\right)\right|=t \geqslant 1$. If $t=1$ and $E_{0} \cap E_{1} \cap E_{\ell}=\emptyset$ this yields a special cycle, and thus the expected number of such hypergraphs in $H_{p}$ is $o(1)$. Otherwise, $t^{\prime}=\left|E_{\ell} \cap\left(E_{0} \cup E_{1}\right)\right| \geqslant 2$. As $\left|E_{1} \cap E_{\ell}\right| \leqslant 1$ we have $s+t^{\prime} \leqslant q+1$. Hence, the expected number of choices for $E_{0}, E_{1}, E_{\ell}$ is

$$
O\left(\sum_{s, t^{\prime}} n p \cdot p^{q-s} \cdot p^{q-t^{\prime}}\right)=O\left(\sum_{s, t^{\prime}} n p^{2 q-\left(s+t^{\prime}\right)+1}\right)=O\left(n p^{q}\right)=o(1)
$$

Assuming that $E$ intersects every edge of a path $\left(E_{1}, \ldots, E_{\ell}\right)$ in at most one vertex, we may define an ordering function $f: E \rightarrow[\ell]$ by $f(v)=\min \left\{i: v \in E_{i}\right\}$ and order the vertices of $E$ by the values of $f$. Observe the shortest sub-path containing the first three vertices $\left\{v_{1}, v_{2}, v_{3}\right\} \subset E$, and denote $t_{1}=f\left(v_{2}\right)-f\left(v_{1}\right)$ and $t_{2}=f\left(v_{3}\right)-f\left(v_{2}\right)$. Let $Y^{\prime}$ count such configurations in $\mathrm{H}$. We have an expected $O(n p)$ many choices for $E$, and $O\left(c_{r}^{t-1} p^{q-2}\right)$ many choices for a path of length $t$ between two fixed vertices. Summing over $t_{1}$ and $t_{2}$ we obtain

$$
\mathbb{E} Y^{\prime}=O\left(\sum_{t_{1} \geqslant 1} \sum_{t_{2} \geqslant 1} n p \cdot c_{r}^{t_{1}-1} p^{q-2} \cdot c_{r}^{t_{2}-1} p^{q-2}\right)=O\left(n p^{2 q-3}\right)=O\left(p^{q-2}\right)=o(1) .
$$

Thus,

$$
\mathbb{E} Y=o(1)
$$

Finally, let $Z$ be the random variable counting the number of copies of the reduced Fano plane in $H_{p}$. First, we show that there are $O\left(n^{2}\right)$ copies of the reduced Fano plane in $H$. Suppose that $\left\{x_{1}, x_{2}, x_{3}\right\}$ is an edge in $H$, since there are $O\left(n^{2}\right)$ choices for an arithmetic progression of length three it suffices to show that there are at most $O(1)$ many choices for $x_{4}, x_{5}, x_{6}$ such that $\left\{x_{1}, \ldots, x_{6}\right\}$ induce a copy of the reduced Fano plane. Denote $\vec{v}=\left(x_{1}, x_{2}, x_{3}\right)$ and $\vec{x}=\left(x_{4}, x_{5}, x_{6}\right)$. Since every pair of vertices in $\left\{x_{4}, x_{5}, x_{6}\right\}$ form a 
3-AP with one vertex in $\left\{x_{1}, x_{2}, x_{3}\right\}$, we obtain the following family of equations:

$$
A \cdot \vec{x}=\vec{v} \quad \text { for } \quad A=\left(\begin{array}{ccc}
a & b & 0 \\
0 & c & d \\
e & 0 & f
\end{array}\right) \quad \text { with } \quad a, b, c, d, e, f \in\left\{-1, \frac{1}{2}, 2\right\} \text {. }
$$

Since $\operatorname{det}(A)=a c f+b d e \neq 0$ for all choices of $A$ we obtain that $A$ is invertible, and therefore fixing $\vec{v}$ determines $\vec{x}$. Since the reduced Fano plane contains six vetices we obtain that the expected number of such configurations is $O\left(n^{2} p^{6}\right)=O\left(n^{2-\frac{6}{2}}\right)=O\left(\frac{1}{n}\right)=$ $o(1)$.

Thus, by Markov's inequality $\mathbb{P}(U=W=X=Y=Z=0) \rightarrow 1$ as $n \rightarrow \infty$ completing the proof.

With the two lemmas in hand, the proof of Theorem 14 is immediate.

Proof of Theorem 14. Let $H$ be the hypergraph with vertex set $V(H)=[n]_{p}$, whose edge set is the set of $q$-term arithmetic progressions. By Lemma 19, $H$ a.a.s. contains no 2-blocking hypergraph. Therefore, by Lemma $17, H$ is 2-colorable.

\section{The asymmetric case 1-statement}

In this section, we prove the following result:

Theorem 20. For every $r \geqslant 2$ and $q_{1}>q_{2} \geqslant \cdots \geqslant q_{r} \in \mathbb{N}, q_{r} \geqslant 3$, there exists $C>0$ such that the following holds:

If $p \geqslant C n^{-\frac{q_{2}}{q_{1}\left(q_{2}-1\right)}}$ then a.a.s. for every $r$-coloring of $[n]_{p}$ there is some $i$ such that there exists a monochromatic $q_{i}$-AP colored $i$.

We begin by describing a rough outline of the proof, which follows similar ideas to [7]. We say that a coloring is proper if it contains no monochromatic $q_{i}$-AP colored $i$; we wish to prove that a.a.s. $[n]_{p}$ admits no such coloring. First, we reduce the problem by showing that every proper coloring may be modified to yield a "good" coloring where, additionally, every element not in a $q_{1}$-AP is colored 1 . Thus, it would suffice to show that no such good coloring exists.

Using The Hypergraph Container Lemma we obtain a set of containers $\mathcal{G}$ for the $q_{2^{-}}$ AP-free subsets of $[n]$. Supposing for contradiction that a good coloring exists, we fix one arbitrary such coloring along with containers $G_{2}, \ldots, G_{r} \in \mathcal{G}$ for all the color classes but the first.

For $i \geqslant 2$, we denote the set of elements colored $i$ by $I_{i}$. We denote the remainder set $I_{1}=[n]_{p} \backslash\left(I_{2} \cup \cdots \cup I_{r}\right)$. Since, by Lemma 12, $[n] \backslash\left(G_{2} \cup \cdots \cup G_{r}\right)$ contains at least $\epsilon n^{2}$ many arithmetic progressions of length $q_{1}$, the set of elements colored 1 is unlikely to be $q_{1}$-AP-free. Indeed, in Lemma 21 , we show that the probability that the set of elements colored 1 contains no $q_{1}$-AP is exponentially small in $n^{2} p^{q_{1}}$.

Note, that the existence of a good coloring implies two events: First, the signature sets for the containers $G_{2}, \ldots, G_{r}$ must all be covered by $q_{1}$-APs in $[n]_{p}$, and second, the 
remainder set $I_{1}$ must not contain a single $q_{1}$-AP. Using Harris's inequality, we show that these two events are negatively correlated.

Finally, we apply a union bound over all possible choices for the tuple of containers $\left(G_{2}, \ldots, G_{r}\right)$, by iterating over their signature sets $S_{2}, \ldots, S_{r}$. A critical part of the union bound argument is Lemma 23, which roughly states that if $p=\Theta\left(n^{-\frac{q_{2}}{q_{1}\left(q_{2}-1\right)}}\right)$ then typically $q_{1}$-APs rarely intersect, and therefore most of $S=\bigcup S_{i}$ is covered by isolated $q_{1}$-APs in $[n]_{p}$.

We begin by proving the following lemmas:

Lemma 21. Suppose $\mathcal{A}$ is a collection of $\Omega\left(n^{2}\right) q$-APs in $[n]$, and $n p^{q-1} \ll 1$. Then,

$$
\mathbb{P}\left([n]_{p} \text { does not contain any member of } \mathcal{A}\right) \leqslant \exp \left(-\Omega\left(n^{2} p^{q}\right)\right) \text {. }
$$

Proof. Enumerate the elements of $\mathcal{A}=\left\{E_{i}: i \in I\right\}$. For each $i \in I$, let $X_{i}$ be the indicator random variable for the event $E_{i} \subset[n]_{p}$ and let $X=\sum X_{i}$. Observe that

$$
\begin{aligned}
& \mu=\mathbb{E} X=\Omega\left(n^{2} p^{q}\right), \\
& \Delta=\sum_{\substack{i \neq j \\
E_{i} \cap E_{j} \neq \emptyset}} \mathbb{P}\left(E_{i} \cup E_{j} \subset[n]_{p}\right)=\sum_{i \in I} \sum_{1 \leqslant k \leqslant q-1} \sum_{\substack{j \in I \\
\left|E_{i} \cap E_{j}\right|=k}} p^{2 q-k} .
\end{aligned}
$$

Note that for a fixed $i$ and $k>1$ there are only at most $q^{2}=O(1)$ many $j$ s such that $\left|E_{i} \cap E_{j}\right|=k$, and for $k=1$ there at most $O(n)$ such $j$ s. For a fixed $i$ this implies,

$$
\sum_{1 \leqslant k \leqslant q-1} \sum_{\substack{j \in I \\\left|E_{i} \cap E_{j}\right|=k}} p^{2 q-k}=O\left(n p^{2 q-1}+\sum_{2 \leqslant k \leqslant q-1} p^{2 q-k}\right)=O\left(n p^{2 q-1}+p^{q+1}\right),
$$

and thus, as $|\mathcal{A}|=O\left(n^{2}\right)$,

$$
\Delta=O\left(n^{3} p^{2 q-1}+n^{2} p^{q+1}\right) .
$$

Moreover, by our assumption that $n p^{q-1} \ll 1$ we have

$$
n^{3} p^{2 q-1}+n^{2} p^{q+1} \ll n^{2} p^{q} .
$$

Hence, by Janson's inequality,

$$
\mathbb{P}(X=0) \leqslant \exp \left(-\mu+\frac{\Delta}{2}\right)=\exp (-\Omega(\mu))=\exp \left(-\Omega\left(n^{2} p^{q}\right)\right)
$$

concluding the proof.

Definition 22. We say that a $q_{1}-\mathrm{AP}$ in $[n]_{p}$ is isolated, if it does not intersect any other $q_{1}-\mathrm{AP}$ in $[n]_{p}$. We define the following random variables:

$$
\begin{aligned}
Q & =\bigcup\left\{A \subset[n]_{p}: A \text { is a } q_{1}-\mathrm{AP}\right\} \\
Q_{I} & =\bigcup\left\{A \subset[n]_{p}: A \text { is an isolated } q_{1}-\mathrm{AP}\right\} .
\end{aligned}
$$

Let $\delta=\frac{\min \left\{q_{2}, q_{1}-q_{2}\right\}}{2 q_{1}\left(q_{2}-1\right)}$. If $\left|Q \backslash Q_{I}\right|<n^{1-\frac{1}{q_{2}-1}-\delta}$ we say that $Q$ is mostly independent. 
Lemma 23. If $p=O\left(n^{-\frac{q_{2}}{q_{1}\left(q_{2}-1\right)}}\right)$ then $Q$ is mostly independent a.a.s.

Proof. First we compute the expected number of sets that are a union of two intersecting $q_{1}$-APs. First, since $\frac{1}{q_{2}-1}-\frac{q_{2}}{q_{1}\left(q_{2}-1\right)}=\frac{q_{1}-q_{2}}{q_{1}\left(q_{2}-1\right)} \geqslant 2 \delta$,

$\mathbb{E}\left(\#\right.$ pairs of $q_{1}$-APs sharing exactly one element $)=O\left(n^{2} \cdot n \cdot p^{2 q_{1}-1}\right)$

$$
=O\left(n^{1-\frac{2}{q_{2}-1}+\frac{q_{2}}{q_{1}\left(q_{2}-1\right)}}\right)=O\left(n^{1-\frac{1}{q_{2}-1}-2 \delta}\right) .
$$

Second, for every $1<m<q_{1}$

$$
\begin{aligned}
\mathbb{E}\left(\# \text { pairs of } q_{1} \text {-APs sharing exactly } m \text { elements }\right) & =O\left(n^{2} \cdot p^{2 q_{1}-m}\right) \\
& =O\left(n^{-\frac{2}{q_{2}-1}+\frac{m q_{2}}{q_{1}\left(q_{2}-1\right)}}\right) .
\end{aligned}
$$

Since $\frac{m q_{2}}{q_{1}\left(q_{2}-1\right)} \leqslant \frac{\left(q_{1}-1\right) q_{2}}{q_{1}\left(q_{2}-1\right)}=1+\frac{1}{q_{2}-1}-\frac{q_{2}}{q_{1}\left(q_{2}-1\right)} \leqslant 1+\frac{1}{q_{2}-1}-2 \delta$,

$$
\mathbb{E}\left(\# \text { pairs of } q_{1} \text {-APs sharing } m \text { elements }\right)=O\left(n^{1-\frac{1}{q_{2}-1}-2 \delta}\right) .
$$

In particular, since $\left|Q \backslash Q_{I}\right| \leqslant|\{(A, B): A \cap B \neq \emptyset, A, B \in Q\}|$,

$$
\mathbb{E}\left|Q \backslash Q_{I}\right| \leqslant O\left(n^{1-\frac{1}{q_{2}-1}-2 \delta}\right) .
$$

Finally, we obtain from Markov's inequality,

$$
\mathbb{P}\left(\left|Q \backslash Q_{I}\right| \geqslant n^{1-\frac{1}{q_{2}-1}-\delta}\right) \leqslant O\left(\frac{n^{1-\frac{1}{q_{2}-1}-2 \delta}}{n^{1-\frac{1}{q_{2}-1}-\delta}}\right)=O\left(n^{-\delta}\right)=o(1)
$$

Hence, a.a.s.

$$
\left|Q \backslash Q_{I}\right|<n^{1-\frac{1}{q_{2}-1}-\delta}
$$

We are now ready to prove Theorem 20.

Proof of Theorem 20. First, since $p$ depends only on $q_{1}$ and $q_{2}$, we may assume that $q_{2}=q_{3}=\cdots=q_{r}$. We say that a coloring is proper if it contains no monochromatic $q_{i}$-AP colored $i$; we wish to prove that a.a.s. $[n]_{p}$ admits no such coloring.

By our assumption, $p \geqslant C n^{-\frac{q_{2}}{q_{1}\left(q_{2}-1\right)}}$ for some sufficiently large $C>0$. Since not admitting a proper coloring is an increasing event, without loss of generality we may assume that $p=C n^{-\frac{q_{2}}{q_{1}\left(q_{2}-1\right)}}$.

Note that the elements of the set $\left\{a \in[n]_{p}: a\right.$ does not belong to a $\left.q_{1}-\mathrm{AP}\right\}$ may all be recolored 1 for any proper coloring of $[n]_{p}$, without creating a monochromatic $q_{1}$-AP. We say that such a proper coloring is a "good" coloring. Since, by recoloring, the existence of a proper coloring implies the existence of a good coloring it suffices to show that no good coloring exists.

Suppose that there is such a coloring; then for each $i \in[r]$, the set $I_{i}$ of elements colored $i$ contains no $q_{i}$-APs. By The Hypergraph Container Lemma for every $\epsilon>0$ and 
every $i \geqslant 2$ there exist $S_{i} \subset I_{i} \subset G_{i}$ with $\left|S_{i}\right| \leqslant s_{\max }=O\left(n^{1-\frac{1}{q_{2}-1}}\right)$ and such that $G_{i}$, which depends only on $S_{i}$, contains at most $\epsilon n^{2}$ many $q_{2}$-APs (the implicit constant in the definition of $s_{\max }$ may depend on $\epsilon$ and $q_{2}$ ). By our assumption,

$$
I_{1}=[n]_{p} \backslash\left(I_{2} \cup \cdots \cup I_{r}\right) \supset[n]_{p} \backslash\left(G_{2} \cup \cdots \cup G_{r}\right)=[n]_{p} \cap\left([n] \backslash\left(G_{2} \cup \cdots \cup G_{r}\right)\right) .
$$

In particular,

$$
I_{1} \text { contains no } q_{1} \text {-APs } \Longrightarrow[n]_{p} \cap\left([n] \backslash\left(G_{2} \cup \cdots \cup G_{r}\right)\right) \text { contains no } q_{1} \text {-APs. }
$$

For brevity, we write $A_{\left(S_{2}, \ldots, S_{r}\right)}=[n]_{p} \cap\left([n] \backslash\left(G_{2} \cup \cdots \cup G_{r}\right)\right)$, as $G_{i}$ depends only on $S_{i}$. Since $q_{1}>q_{2}$, the number of $q_{1}$-APs in any set of integers is at most as large as the number of $q_{2}$-APs. Therefore, for every choice of $\left(S_{2}, \ldots, S_{r}\right)$, Lemma 12 implies that $[n] \backslash\left(G_{2} \cup \cdots \cup G_{r}\right)$ contains at least $\epsilon n^{2}$ many $q_{1}$-APs. Hence, by Lemma 21

$$
\mathbb{P}\left(A_{\left(S_{2}, \ldots, S_{r}\right)} \text { contains no } q_{1}-\mathrm{AP}\right) \leqslant e^{-D_{1} n^{2} p^{q_{1}}}
$$

for some constant $D_{1}>0$ which depends only on $\epsilon$ and $q_{1}$.

Let $Q$ and $Q_{I}$ be the variables defined in Definition 22. Suppose $S=\cup_{i=2}^{r} S_{i}$ is covered by elements of $Q$; we fix a largest subset of $S$ that is covered by pairwise-disjoint arithmetic progressions of length $q_{1}$ in $[n]_{p}$ and denote it $S^{\prime}$. Since $S \backslash S^{\prime} \subset S \cap\left(Q \backslash Q_{I}\right)$, if $Q$ is mostly independent then $\left|S \backslash S^{\prime}\right|<n^{1-\frac{1}{q_{2}-1}-\delta}$. Thus, if a good coloring exists and $Q$ is mostly independent then there exists some choice of $\left(S_{2}, \ldots, S_{r}\right)$ such that

1. $S$ is covered by $q_{1}-\mathrm{APs}$ in $[n]_{p}$.

2. $\left|S \backslash S^{\prime}\right|<n^{1-\frac{1}{q_{2}-1}-\delta}$.

3. $A_{\left(S_{2}, \ldots, S_{r}\right)}$ contains no $q_{1}-\mathrm{AP}$.

For shorthand, we say that $S$ is "well-covered" if it satisfies conditions 1 and 2.

Let $P=\mathbb{P}\left([n]_{p}\right.$ admits a "good" coloring). We wish to show that $P=o(1)$. We first note $P$ may be bounded by the sum of probabilities of two other events: either $Q$ is not mostly independent or there exists a tuple $\left(S_{2}, \ldots, S_{r}\right)$ such that the above three events hold. Since by Lemma 23 the probability that $Q$ is not mostly independent is $o(1)$ we obtain

$$
P \leqslant o(1)+\sum_{\left(S_{2}, \ldots, S_{r}\right)} \mathbb{P}\left(S \text { is well-covered } \wedge A_{\left(S_{2}, \ldots, S_{r}\right)} \text { contains no } q_{1} \text {-AP }\right) .
$$

Note, that the event " $S$ is well-covered" is increasing, while the event " $A_{\left(S_{2}, \ldots, S_{r}\right)}$ contains no $q_{1}-\mathrm{AP} "$ is decreasing. Therefore by Harris's inequality we obtain

$$
\begin{aligned}
P & \leqslant o(1)+\sum_{\left(S_{2}, \ldots, S_{r}\right)} \mathbb{P}(S \text { is well-covered }) \cdot \mathbb{P}\left(A_{\left(S_{2}, \ldots, S_{r}\right)} \text { contains no } q_{1} \text {-AP }\right) \\
& \leqslant o(1)+\sum_{\left(S_{2}, \ldots, S_{r}\right)} \mathbb{P}(S \text { is well-covered }) \cdot e^{-D_{1} n^{2} p^{q_{1}}} .
\end{aligned}
$$


Our goal is now to obtain a bound on

$$
\sum_{\left(S_{2}, \ldots, S_{r}\right)} \mathbb{P}(S \text { is well-covered })
$$

Let $\mathcal{C}(A)$ denote the event " $A$ is covered by pairwise-disjoint $q_{1}$-APs". Since there are at most $r^{\left|S \backslash S^{\prime}\right|}$ many ways to distribute the elements of $S \backslash S^{\prime}$ to $S_{2}, \ldots, S_{r}$, we obtain

$$
\sum_{\left(S_{2}, \ldots, S_{r}\right)} \mathbb{P}(S \text { is well-covered }) \leqslant \sum_{t=0}^{n^{1-\frac{1}{q_{2}-1}-\delta}}\left(\begin{array}{l}
n \\
t
\end{array}\right) r^{t} \cdot \sum_{\left(S_{2}^{\prime}, \ldots, S_{r}^{\prime}\right)} \mathbb{P}\left(\mathcal{C}\left(S^{\prime}\right)\right)
$$

where the second sum ranges over all $(r-1)$-tuples of sets $\left(S_{2}^{\prime}, \ldots, S_{r}^{\prime}\right)$ satisfying $\left|S_{i}^{\prime}\right| \leqslant s_{\max }$ for each $i$ and $S^{\prime}=\cup_{i=2}^{r} S_{i}^{\prime}$; which we may bound from above by

$$
O\left(e^{3 \log n \cdot n^{1-\frac{1}{q_{2}-1}-\delta}}\right) \cdot \sum_{\left(S_{2}^{\prime}, \ldots, S_{r}^{\prime}\right)} \mathbb{P}\left(\mathcal{C}\left(S^{\prime}\right)\right) .
$$

We now move on to bound

$$
\sum_{\left(S_{2}^{\prime}, \ldots, S_{r}^{\prime}\right)} \mathbb{P}\left(\mathcal{C}\left(S^{\prime}\right)\right)
$$

Suppose $S^{\prime}=\cup_{i=2}^{r} S_{i}^{\prime}$ is fixed, then there are $(r-1)^{\left|S^{\prime}\right|}$ many ways to distribute its elements into $r-1$ different subsets; hence,

$$
\sum_{\left(S_{2}^{\prime}, \ldots, S_{r}^{\prime}\right)} \mathbb{P}\left(\mathcal{C}\left(S^{\prime}\right)\right) \leqslant O\left(2^{r s_{\max }}\right) \sum_{\left|S^{\prime}\right|<r s_{\max }} \mathbb{P}\left(\mathcal{C}\left(S^{\prime}\right)\right)
$$

We note that $\sum_{\left|S^{\prime}\right|<r s_{\max }} \mathbb{P}\left(\mathcal{C}\left(S^{\prime}\right)\right)$ is simply the expected number of sets of size at most $r s_{\max }$ that are covered by pairwise-disjoint $q_{1}$-APs. This, in turn, may be bounded from above by

$$
\sum_{N \leqslant r s_{\max }} \sum_{s<r s_{\max }}\left(\begin{array}{c}
q_{1} N \\
s
\end{array}\right) \mathbb{E}\left(\# \text { collections of } N \text { pairwise-disjoint } q_{1} \text {-APs in }[n]_{p}\right) .
$$

Since the expected number of choices for collections of $N$ pairwise-disjoint $q_{1}$-APs in $[n]_{p}$ is at most $\frac{\left(n^{2} p^{q_{1}}\right)^{N}}{N !}$, we conclude

$$
\begin{aligned}
\sum_{\left|S^{\prime}\right|<r s_{\max }} \mathbb{P}\left(\mathcal{C}\left(S^{\prime}\right)\right) & \leqslant \sum_{N \leqslant r s_{\max }} \sum_{s<r s_{\max }} \frac{\left(n^{2} p^{q_{1}}\right)^{N}}{N !}\left(\begin{array}{c}
q_{1} N \\
s
\end{array}\right) \\
& \leqslant \sum_{N \leqslant r s_{\max }} \frac{\left(n^{2} p^{q_{1}}\right)^{N}}{N !} 2^{q_{1} N} \\
& \leqslant \sum_{N \leqslant r s_{\max }}\left(\frac{e 2^{q_{1}} n^{2} p^{q_{1}}}{N}\right)^{N} .
\end{aligned}
$$


Note, that $N \leqslant r s_{\max } \leqslant D_{2}^{\prime} n^{1-\frac{1}{q_{2}-1}}$ for some $D_{2}^{\prime}>0$ which does not depend on $C$. Since $x \rightarrow\left(\frac{e a}{x}\right)^{x}$ grows for $x \leqslant a$ and $n^{2} p_{1}^{q}=C^{q_{1}} n^{1-\frac{1}{q_{2}-1}}$, for $C$ large enough we have

$$
\left(\frac{e 2^{q_{1}} n^{2} p^{q_{1}}}{N}\right)^{N} \leqslant\left(\frac{(e 2 C)^{q_{1}}}{D_{2}^{\prime}}\right)^{D_{2}^{\prime} n^{1-\frac{1}{q_{2}-1}}} \leqslant e^{D_{2}^{\prime} q_{1} \log (2 e C) n^{1-\frac{1}{q_{2}-1}}} .
$$

Hence,

$$
\sum_{N<r s_{\max }}\left(\frac{e 2^{q_{1}} n^{2} p^{q_{1}}}{N}\right)^{N} \leqslant \sum_{N<r s_{\max }} e^{D_{2}^{\prime} q_{1} \log (2 e C) n^{1-\frac{1}{q_{2}-1}}} \leqslant e^{O\left(\log C n^{1-\frac{1}{q_{2}-1}}\right)} .
$$

Finally we obtain,

$$
\begin{aligned}
\sum_{\left(S_{2}, \ldots, S_{r}\right)} \mathbb{P}(S \text { is well-covered }) & \leqslant O\left(e^{O\left(n^{1-\frac{1}{q_{2}-1}} \log C+\log n \cdot n^{1-\frac{1}{q_{2}-1}-\delta}\right)}\right) \\
& \leqslant e^{D_{2} \log C n^{1-\frac{1}{q_{2}-1}}}
\end{aligned}
$$

for some constant $D_{2}>0$ which does not depend on C.

Therefore, for $C$ large enough,

$$
\begin{aligned}
P & \leqslant o(1)+\mathbb{P}(S \text { is well-covered }) \cdot e^{-D_{1} n^{2} p^{q_{1}}} \\
& =o(1)+O\left(e^{D_{2} \log C n^{1-\frac{1}{q_{2}-1}}}\right) \cdot O\left(e^{-D_{1} C^{q_{1}} n^{1-\frac{1}{q_{2}-1}}}\right) \rightarrow 0
\end{aligned}
$$

\section{The asymmetric case 0 -statement}

In this section, we show that for any integers $q_{1}>q_{2}$ there exists a sufficiently small positive $c>0$ such that if $p=c \cdot n^{-\frac{q_{2}}{q_{1}\left(q_{2}-1\right)}}$ the elements of $[n]_{p}$ can a.a.s. be colored red/blue without a monochromatic $q_{1}$-AP colored red or a monochromatic $q_{2}$-AP colored blue. We note that this is sufficient for the 0 -statement for any other number of colors. We begin by making several definitions that will assist us in stating the results of this section in the language of hypergraphs.

Definition 24. Throughout this section we will deal with hypergraphs with edges of two possible cardinalities $q_{1}$ and $q_{2}$, we call such hypergraphs $\left(q_{1}, q_{2}\right)$-uniform. We will refer to edges as long or short edges, depending on their cardinalities. We say that a $\left(q_{1}, q_{2}\right)$ uniform hypergraph is asymmetrically-2-colorable if its vertices can be colored red/blue with no long edge colored red, and no short edge colored blue.

Definition 25. Let $H\left(n, q_{1}, q_{2}\right)$ be the hypergraph with vertex set $V(H)=[n]$, whose edge set is the set of arithmetic progressions of lengths $q_{1}$ and $q_{2}$. We denote by $H\left(n, q_{1}, q_{2}, p\right)$ the random subhypergraph of $H\left(n, q_{1}, q_{2}\right)$ induced by $[n]_{p}$.

With these definitions in hand, we are ready to state this section's main result: 
Theorem 26 (Asymmetric 0-statement). For any integers $q_{1}>q_{2} \geqslant 3$, there exists $c>0$ such that for $p \leqslant c \cdot n^{-\frac{q_{2}}{q_{1}\left(q_{2}-1\right)}}, H\left(n, q_{1}, q_{2}, p\right)$ is asymmetrically-2-colorable a.a.s.

The proof we present here is similar in nature to the proof by Rödl and Ruciński [20] of the (symmetric) random Rado partition theorem and consists of two main lemmas. First, in Lemma 33, we show that a $\left(q_{1}, q_{2}\right)$-uniform hypergraph is asymmetrically-2-colorable unless it contains a member of a small family of hypergraphs which we call 2-blocking. Then, Lemma 34 states that those hypergraphs a.a.s. do not appear in $H\left(n, q_{1}, q_{2}, p\right)$. We begin by making several definitions.

Definition 27. We say that an edge $E=\left\{a_{1}, \ldots, a_{q}\right\}$ has a cover if there are edges $E_{1}, \ldots, E_{q}$ such that $E \cap E_{i}=\left\{a_{i}\right\}$ and $\left|E_{i}\right| \neq|E|$ for all $i \in[q]$. We say that a cover for an edge is simple if $E_{i} \cap\left(\bigcup_{j \neq i} E_{j}\right)=\emptyset$ for all $i \in[q]$. If every edge in a hypergraph $H$ has a cover, we say $H$ is covered.

Definition 28. A simple path of length $\ell$ is a hypergraph consisting of short edges $E_{1}, \ldots, E_{\ell}$, and covering long edges $E_{1,1}, \ldots, E_{1, q_{2}}, E_{2,1}, \ldots, E_{\ell, q_{2}}$ such that $E_{i, 1}, \ldots, E_{i, q_{2}}$ cover $E_{i}$ and such that:

1. $E_{i+1,1}=E_{i, q_{2}}$ for every $i<\ell$,

2. no two edges of the same cardinality intersect.

A simple path of length one is called a block; thus, a simple path consists of blocks, such that every pair of consecutive blocks share a long edge. For convenience, we refer to a single long edge as a simple path of length zero.

Definition 29. We say that a simple path $P$ of length $\ell$ has a saw if for every $v_{i} \in E_{1,1} \backslash E_{1}$ there exists a short edge $S_{i}$ such that $S_{i} \cap E_{1,1}=\left\{v_{i}\right\}$ and $\left|S_{i} \cap V(P)\right|=2$. We call the edges $S_{i}$ the saw edges for $P$.

Definition 30. We say that a simple path $P$ of length $\ell$ is spoiled if there exists an edge $E \notin E(P)$ such that $|E \cap V(P)| \geqslant 3$ and $E \cap E_{\ell, q_{2}}=\{v\}$ for some $v \notin E_{\ell}$.

Definition 31. We say that a simple path $P$ of length $\ell$ has a spoiled extension, if there exists a short edge $E_{\ell+1}$ along with a simple cover $E_{\ell+1,1}, \ldots, E_{\ell+1, q_{2}}$, such that $E_{\ell+1} \cap V(P)=\{v\}$ for some $v \in E_{\ell, q_{2}} \backslash E_{\ell}, E_{\ell+1,1}=E_{\ell, q_{2}}$ and there exists $i \in\left\{2, \ldots, q_{2}\right\}$ such that $E_{\ell+1, i} \cap V(P) \neq \emptyset$.

Definition 32. We say that a $\left(q_{1}, q_{2}\right)$-uniform hypergraph is 2-blocking if it is one of the following:

1. A short edge with a non-simple cover.

2. A spoiled simple path.

3. A simple path with a saw. 


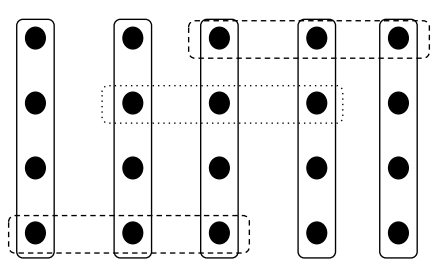

(a) A spoiled simple path of length 2 , with the spoiling edge dotted.

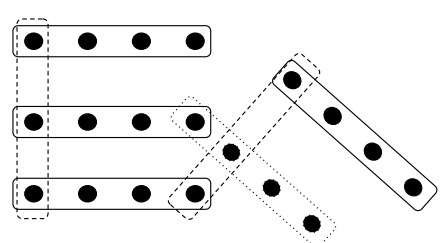

(b) A block with a spoiled extension, with the spoiling long edge dotted.

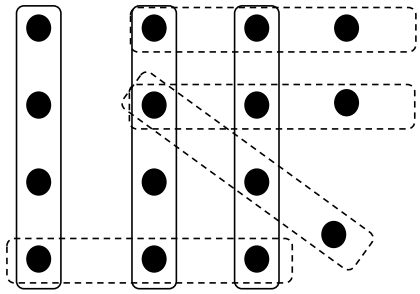

(c) A path of length one with a saw.

Figure 2: Examples of 2-blocking hypergraphs for $q_{1}=4, q_{2}=3$.

4. A simple path with a spoiled extension.

Lemma 33 (The deterministic lemma). Let $q_{1}>q_{2} \geqslant 3$ be some integers, and let $H$ be a $\left(q_{1}, q_{2}\right)$-uniform hypergraph which is not asymmetrically-2-colorable. Then $H$ contains a 2-blocking hypergraph.

Lemma 34 (The probabilistic lemma). Let $H=H\left(n, q_{1}, q_{2}, p\right)$, let $c$ be a sufficiently small positive constant, and let $p=c \cdot n^{-\frac{q_{2}}{q_{1}\left(q_{2}-1\right)}}$. Then a.a.s. $H$ contains no 2-blocking hypergraph.

Theorem 26 follows immediately from these two lemmas. Most of this section deals with proving the probabilistic lemma, using a first-moment argument over several random variables. But first, we begin by proving the deterministic part of the theorem.

Proof of The deterministic lemma. We say that a hypergraph is edge-critical if it is not asymmetrically-2-colorable, but any proper subhypergraph is. We may assume that $H$ is edge-critical; otherwise, we replace it with an edge-critical subhypergraph. We begin by showing that every edge critical hypergraph is covered.

Claim 35. If $H$ is an edge-critical hypergraph, then for every edge $E \in H$ and for every vertex $v \in E$ there is an edge $E^{\prime} \in H$ such that $E \cap E^{\prime}=\{v\}$ and $|E| \neq\left|E^{\prime}\right|$; in other words, $H$ is covered.

Proof. Let $H$ be edge-critical, and suppose that there are an edge $E \in H$ and a vertex $v \in E$, such that every edge $E^{\prime}$ of the other cardinality that contains $v$ also contains another vertex of $E$. By the edge-criticality, $H$ can be colored red/blue in such a way that only $E$ violates the coloring condition. Without loss of generality, assume $E$ is long (and colored red). Now, by changing the color of $v$ to blue, $E$ no longer violates the coloring condition, and neither does any short edge that contains $v$, contradicting the fact that $H$ is not asymmetrically-2-colorable.

If there exists a short edge with a non-simple cover, then we are done, so we may assume all short edges have simple covers. Let $P$ be a longest simple path, and let $\ell \geqslant 1$ 
be its length. We observe $E=E_{\ell, q_{2}}$; since $E$ is covered by short edges, we have short edges $S_{i}$ such that $S_{i} \cap E=\left\{v_{i}\right\}$ for every $v_{i} \in\left\{v_{2}, \ldots, v_{q_{1}}\right\}=E \backslash E_{\ell}$.

Suppose first that there exists $S_{i}$ such that $S_{i} \cap V(P)=\left\{v_{i}\right\}$ and observe $\left\{s_{2}, \ldots, s_{q_{2}}\right\}=$ $S_{i} \backslash E$. By the previous claim, we have a long covering edge for every $s_{i}$, and by the maximality of $P$ we obtain that for every simple cover for $S_{i}$ there exists a covering edge $L$ that intersects $P$. Thus, we obtain that $S_{i}$ forms a spoiled extension to $P$.

If, on the other hand, no $S_{i}$ intersects $P$ in exactly a single vertex, we obtain one of two cases: If there exists some $i$ such that $\left|S_{i} \cap V(P)\right| \geqslant 3$, then since $\left|S_{i} \cap E\right|=1$ we obtain that $S_{i}$ is a spoiling edge for $P$. Otherwise, we have $\left|S_{i} \cap V(P)\right|=2$ for all $i$ and thus obtain a path with a saw, completing the proof.

The rest of this section deals with proving the probabilistic portion of Theorem 26 . We will begin by proving upper bounds on the number of copies of several hypergraphs in $H\left(n, q_{1}, q_{2}\right)$ and showing that a.a.s. all short edges have simple covers. We will then prove an upper bound on the number of simple paths of arbitrary lengths. Finally, with the above results in hand, we will turn to prove Lemma 34.

Lemma 36. Let $x, y \in[n]$ be distinct integers. Then, the number of choices for $a, b \in[n]$ such that there are $q$-APs that contain $\{x, a, b\}$ and $\{y, a, b\}$ is $O(1)$.

Proof. Suppose that $a, b$ are contained in a $q$-AP along with $x$, then there exists $t_{1} \in \mathbb{Q}$ such that $x-a=t_{1}(b-a)$, thus, $x=\left(1-t_{1}\right) a+t_{1} b$. Moreover, $t_{1}=\frac{r_{1}}{r_{2}}$ for $r_{1}, r_{2} \in$ $\{-q,-q+1, \ldots, q\}$. The same also holds for $y$ with another constant $t_{2}$. We obtain the following system of linear equations:

$$
\left(\begin{array}{ll}
1-t_{1} & t_{1} \\
1-t_{2} & t_{2}
\end{array}\right)\left(\begin{array}{l}
a \\
b
\end{array}\right)=\left(\begin{array}{l}
x \\
y
\end{array}\right)
$$

Since the determinant of the above matrix is $t_{2}-t_{1}$, we obtain that as long as $t_{1} \neq t_{2}$ there is only a single solution to $(\star)$. Since $x \neq y$ implies $t_{1} \neq t_{2}$ and there are at most $O\left(q^{2}\right)=O(1)$ choices for $t_{1}$ and $t_{2}$, there are only $O(1)$ many choices for $a, b$.

In Lemma 39 we show that a short edge with its $q_{2}$ covering long edges must contain almost $2 q_{1}$ vertices. We will then use this lemma to show that non-simple covers are unlikely. We will require the following two elementary lemmas.

Lemma 37. Let $E_{1}, E_{2}$ be two arithmetic progressions of length $q_{1}$, with common differences $d_{1}$ and $d_{2}$. If $d_{1}<d_{2}$, then $\left|E_{1} \cap E_{2}\right| \leqslant\left\lceil q_{1} \cdot \frac{\operatorname{gcd}\left(d_{1}, d_{2}\right)}{d_{2}}\right\rceil$.

Proof. Let $A_{1}$ and $A_{2}$ be the infinite arithmetic progressions containing $E_{1}$ and $E_{2}$ respectively. We obtain that $A=A_{1} \cap A_{2}$ is either empty or an infinite arithmetic progression with common difference $\operatorname{lcm}\left(d_{1}, d_{2}\right)$, thus $A \cap A_{1}$ contains every $\frac{\operatorname{lcm}\left(d_{1}, d_{2}\right)}{d_{1}}$-th element of $A_{1}$. Therefore, a subsequence of length $q_{1}$ in $A_{1}$ contains at most $\left\lceil q_{1} \cdot \frac{d_{1}}{\operatorname{lcm}\left(d_{1}, d_{2}\right)}\right\rceil$ elements of $A$, and the result follows immediately. 
Lemma 38. Let $n>m>0$ be integers, and $A=\left(a_{1}, a_{2}, \ldots, a_{q}\right)$ be an arithmetic progression of length $q>3$ with common difference $m$. If we denote $t=\frac{n}{\operatorname{gcd}(n, m)}$, then $\mid\left\{a \in[n]: \exists i \in[q]\right.$ such that $\left.a \equiv a_{i} \bmod n\right\} \mid=\min \{t, q\}$. Moreover, $a_{i} \equiv a_{i+k t} \bmod n$ for all integers $i$ and $k$ such that $i, i+k t \in[q]$.

Proof. Let $G=\mathbb{Z} / n \mathbb{Z}$ be the additive cyclic group of order $n$. From elementary group theory we know that the order of $m$ in $G$ is $t=\frac{n}{\operatorname{gcd}(n, m)}$. Let $G^{\prime}$ be the cyclic subgroup generated by $m$. Then, the residues of $A$ modulo $n$ are contained in the coset $a_{1}+G^{\prime}$, which has $\left|a_{1}+G^{\prime}\right|=\left|G^{\prime}\right|=t$, completing the proof.

Lemma 39. If $E$ is a short edge and $E_{1}, \ldots, E_{r}$ are a subset of its covering edges, then

$$
\left|E_{1} \cup \cdots \cup E_{r}\right|>2 q_{1}\left(1-\frac{1}{r}\right) .
$$

Proof. Denote $M=\left|E_{1} \cup \cdots \cup E_{r}\right|$ and assume for contradiction that $M \leqslant 2 q_{1}\left(1-\frac{1}{r}\right)$. The case $r \in\{1,2\}$ is trivial, therefore we may assume $r \geqslant 3$. We first show that no three covering edges share the same common difference.

Claim 40. Let $E_{1}, E_{2}, E_{3}$ be covering edges, and assume that all three $q_{1}$-APs have the same common difference, i.e. $E_{z}=\left\{a_{z}+i \cdot d: i \in\left[q_{1}\right]\right\}$. Then,

$$
\left|E_{1} \cup E_{2} \cup E_{3}\right| \geqslant 2 q_{1} \text {. }
$$

Proof. Suppose $\left\{v_{z}\right\}=E_{z} \cap E$ for $z \in\{1,2,3\}$. Without loss of generality assume that $v_{1}<v_{2}<v_{3}$, and that $E_{z} \cap E_{2} \neq \emptyset$ for $z=1,3$, as otherwise $\left|E_{z} \cup E_{2}\right|=2 q_{1}$. Since each covering edge may only contain one vertex of $E$, and $E_{1}$ lies on the same infinite arithmetic progression of difference $d$ as $E_{2}$, we deduce that all the elements of $E_{1}$ must be strictly smaller than $v_{2}$. Similarly, all elements of $E_{3}$ must be strictly larger than $v_{2}$. Hence, $E_{1} \cap E_{3}=\emptyset$; and thus, $\left|E_{1} \cup E_{3}\right|=2 q_{1}$.

We will now show that $r \geqslant 5$. Since $r \geqslant 3$, the previous claim implies that there exists a pair of edges with different common differences; without loss of generality we denote them $E_{1}, E_{2}$. We note that $\left|E_{1} \cap E_{2}\right| \leqslant\left\lceil\frac{q_{1}}{2}\right\rceil$, by Lemma 37. Let $E^{\prime}=E \cap\left(E_{1} \cup \cdots \cup E_{r}\right)$. Since $E_{1}, E_{2}$ are covering edges of $E$, we have

$$
\begin{aligned}
\left|E^{\prime} \cup E_{1} \cup E_{2}\right|=\left|E_{1} \cup E_{2}\right|+\left|E^{\prime}\right|-2 & =\left|E_{1}\right|+\left|E_{2}\right|-\left|E_{1} \cap E_{2}\right|+\left|E^{\prime}\right|-2 \\
& \geqslant q_{1}+\left\lfloor\frac{q_{1}}{2}\right\rfloor+r-2 .
\end{aligned}
$$

Thus, if $r \in\{3,4\}$ we observe that (since $\left\lfloor\frac{q_{1}}{2}\right\rfloor \geqslant \frac{q_{1}}{2}-\frac{1}{2}$ )

$$
q_{1}+\left\lfloor\frac{q_{1}}{2}\right\rfloor+r-2 \geqslant \frac{3}{2} q_{1}+r-\frac{5}{2} \geqslant\left(1-\frac{1}{r}\right) 2 q_{1}+r-\frac{5}{2}>2 q_{1}\left(1-\frac{1}{r}\right)
$$

for all $q_{1}>r$. Finally, we will show that no three arithmetic progressions may have pairwise different common differences. 
Claim 41. Let $E_{1}, E_{2}, E_{3}$ be covering edges, with distinct common differences $d_{1}, d_{2}, d_{3}$ respectively. Then

$$
\left|E_{1} \cup E_{2} \cup E_{3}\right| \geqslant 2 q_{1}-4
$$

and thus $M \geqslant\left|E_{1} \cup E_{2} \cup E_{3}\right|+(r-3) \geqslant 2 q_{1}-2>2 q_{1}\left(1-\frac{1}{r}\right)$.

Proof. Assume for contradiction that $\left|E_{1} \cup E_{2} \cup E_{3}\right|<2 q_{1}-4$. Since

$$
\left|E_{1} \cup E_{2} \cup E_{3}\right| \geqslant 3 q_{1}-\left|E_{1} \cap E_{2}\right|-\left|E_{1} \cap E_{3}\right|-\left|E_{2} \cap E_{3}\right|
$$

at least one pair of edges intersects in more than $\frac{q_{1}}{3}+1$ elements.

Recall that $q_{1}>q_{2} \geqslant r \geqslant 5$. Note that $\frac{q_{1}}{3}+1>\left\lceil\frac{q_{1}}{3}\right\rceil \geqslant\left\lceil\frac{q_{1}}{d}\right\rceil$ for all $d \geqslant 3$ and $q_{1} \geqslant 6$. Thus, by Lemma 37, we have that for some $i, j \in[3]$ such that $d_{i}>d_{j}$ we have $\operatorname{gcd}\left(d_{i}, d_{j}\right)=\frac{d_{i}}{2}$ which can only occur if $d_{i}=2 d_{j}$. Let $k \notin\{i, j\}$. Since

$\left|E_{i} \cup E_{j} \cup E_{k}\right| \geqslant 3 q_{1}-\left\lceil\frac{q_{1}}{2}\right\rceil-\left|E_{i} \cap E_{k}\right|-\left|E_{j} \cap E_{k}\right| \geqslant 3 q_{1}-\frac{q_{1}}{2}-1-\left|E_{i} \cap E_{k}\right|-\left|E_{j} \cap E_{k}\right|$

we obtain that $E_{k}$ must intersect one of the other edges in more than $\frac{q_{1}}{4}+1$ elements. Note that $\frac{q_{1}}{4}+1>\left\lceil\frac{q_{1}}{4}\right\rceil \geqslant\left\lceil\frac{q_{1}}{d}\right\rceil$ for all $d \geqslant 4$ and $q_{1} \geqslant 6$. Again, by Lemma 37, we have that $t \cdot \operatorname{gcd}\left(d_{k}, d_{z}\right)=\max \left(d_{k}, d_{z}\right)$ for some $z \in\{i, j\}$ and $t \in\{2,3\}$.

If we set $d_{j}=d$, we get that $\frac{d_{k}}{d}$ may obtain one of six values: either $\frac{1}{2}$ or 4 for $t=2$ or one of $\frac{1}{3}, \frac{2}{3}, 3,6$ for $t=3$. We note that by Lemma 38, if we observe the residues modulo $d^{\prime}$ of an arithmetic progression of length $q_{1}$ with common difference $d<d^{\prime}$, we obtain that the progression cycles through $\frac{d^{\prime}}{\operatorname{gcd}\left(d, d^{\prime}\right)}$ residues; and thus, it contains at least $\left\lfloor\frac{q_{1} \cdot \operatorname{gcd}\left(d, d^{\prime}\right)}{d^{\prime}}\right\rfloor$ elements of every residue class it encounters. By adjusting the constant $d$ and permuting the indices we obtain that the triplet $\left(d_{1}, d_{2}, d_{3}\right)$ must fall into one of five categories:

1. Assuming $\left(d_{1}, d_{2}, d_{3}\right)=(d, 2 d, 4 d)$, without loss of generality we assume $0 \in E_{3}$ and $d=1$. Then, $E_{3}$ consists of $q_{1}$ elements which satisfy $a \equiv 0 \bmod 4$. If $E_{2}$ contains elements which satisfy $a \equiv 1,3 \bmod 4$, we obtain that $\left|E_{3} \cup E_{2}\right|=2 q_{1}$.

Otherwise, we obtain that at least $\left\lfloor\frac{q_{1}}{2}\right\rfloor$ of the elements in $E_{2}$ satisify $a \equiv 2 \bmod 4$, and at least $2 \cdot\left\lfloor\frac{q_{1}}{4}\right\rfloor$ of the elements in $E_{1}$ satisfy $a \equiv 1,3 \bmod 4$. Thus,

$$
\left|E_{1} \cup E_{2} \cup E_{3}\right| \geqslant q_{1}+\left\lfloor\frac{q_{1}}{2}\right\rfloor+2 \cdot\left\lfloor\frac{q_{1}}{4}\right\rfloor \geqslant 2 q_{1}-2 .
$$

2. Assuming $\left(d_{1}, d_{2}, d_{3}\right)=(d, 2 d, 3 d)$, without loss of generality we assume $0 \in E_{3}$ and $d=1$. Then, $E_{3}$ consists of $q_{1}$ elements which satisfy $a \equiv 0,3 \bmod 6$. Since at least $2\left\lfloor\frac{q_{1}}{3}\right\rfloor$ of the elements in $E_{2}$ satisify $a \equiv 2,4 \bmod 6($ or equivalently $1,5 \bmod 6)$, and at least $2\left\lfloor\frac{q_{1}}{6}\right\rfloor$ of the elements in $E_{1}$ satisfy $a \equiv 1,5 \bmod 6$ (equivalently 2,4 mod 6), we obtain

$$
\left|E_{1} \cup E_{2} \cup E_{3}\right| \geqslant q_{1}+2\left\lfloor\frac{q_{1}}{3}\right\rfloor+2\left\lfloor\frac{q_{1}}{6}\right\rfloor \geqslant 2 q_{1}-3 .
$$


3. Assuming $\left(d_{1}, d_{2}, d_{3}\right)=(d, 2 d, 6 d)$, without loss of generality we assume $0 \in E_{3}$ and $d=1$. Then, $E_{3}$ consists of $q_{1}$ elements which satisfy $a \equiv 0 \bmod 6$. If $E_{2}$ contains elements which satisfy $a \equiv 1,3,5 \bmod 6$ we obtain that $\left|E_{3} \cup E_{2}\right|=2 q_{1}$.

Otherwise, at least $2\left\lfloor\frac{q_{1}}{3}\right\rfloor$ of the elements in $E_{2}$ satisify $a \equiv 2,4 \bmod 6$, and at least $3\left\lfloor\frac{q_{1}}{6}\right\rfloor$ of the elements in $E_{1}$ satisfy $a \equiv 1,3,5 \bmod 6$. Hence,

$$
\left|E_{1} \cup E_{2} \cup E_{3}\right| \geqslant q_{1}+2\left\lfloor\frac{q_{1}}{3}\right\rfloor+3\left\lfloor\frac{q_{1}}{6}\right\rfloor \geqslant 2 q_{1}-4 .
$$

4. Assuming $\left(d_{1}, d_{2}, d_{3}\right)=(d, 3 d, 6 d)$, without loss of generality we assume $0 \in E_{3}$ and $d=1$. Then, $E_{3}$ consists of $q_{1}$ elements which satisfy $a \equiv 0 \bmod 6$. If $E_{2}$ contains elements which satisfy $a \equiv 1,4 \bmod 6$ or $a \equiv 2,5 \bmod 6$ we obtain that $\left|E_{3} \cup E_{2}\right|=2 q_{1}$.

Otherwise, at least $\left\lfloor\frac{q_{1}}{2}\right\rfloor$ of the elements in $E_{2}$ satisify $a \equiv 3 \bmod 6$, and at least $4\left\lfloor\frac{q_{1}}{6}\right\rfloor$ of the elements in $E_{1}$ satisfy $a \equiv 1,2,4,5 \bmod 6$. Therefore,

$$
\left|E_{1} \cup E_{2} \cup E_{3}\right| \geqslant q_{1}+4\left\lfloor\frac{q_{1}}{6}\right\rfloor+\left\lfloor\frac{q_{1}}{2}\right\rfloor \geqslant 2 q_{1}-4 .
$$

5. Assuming $\left(d_{1}, d_{2}, d_{3}\right)=(2 d, 3 d, 6 d)$, without loss of generality we assume $0 \in E_{3}$ and $d=1$. Then, $E_{3}$ consists of $q_{1}$ elements which satisfy $a \equiv 0 \bmod 6$. If $E_{2}$ contains elements which satisfy $a \equiv 1,4 \bmod 6$ or $a \equiv 2,5 \bmod 6$ we obtain that $\left|E_{3} \cup E_{2}\right|=2 q_{1}$.

If $E_{1}$ contains elements which satisfy $a \equiv 1,3,5 \bmod 6$ we obtain that $\left|E_{3} \cup E_{1}\right|=$ $2 q_{1}$.

Otherwise, at least $\left\lfloor\frac{q_{1}}{2}\right\rfloor$ of the elements in $E_{2}$ satisify $a \equiv 3 \bmod 6$, and at least $2\left\lfloor\frac{q_{1}}{3}\right\rfloor$ of the elements in $E_{1}$ satisfy $a \equiv 2,4 \bmod 6$. Thus,

$$
\left|E_{1} \cup E_{2} \cup E_{3}\right| \geqslant q_{1}+2\left\lfloor\frac{q_{1}}{3}\right\rfloor+\left\lfloor\frac{q_{1}}{2}\right\rfloor \geqslant 2 q_{1}-2 .
$$

Finally we obtain that $r \geqslant 5$, no three edges may share a common difference, and there can be no more than two possible common differences which yields a contradiction.

Lemma 42. Let $H^{\prime}$ be a hypergraph consisting of a short edge and its non-simple cover. If $p=O\left(n^{-\frac{q_{2}}{q_{1}\left(q_{2}-1\right)}}\right)$, then $\mathbb{P}\left(H^{\prime} \subset H\left(n, q_{1}, q_{2}, p\right)\right)=o(1)$.

Proof. The following computations are used several times throughout the proof:

$$
p^{q_{1}-1}=O\left(n^{-\frac{q_{2}}{q_{2}-1}+\frac{q_{2}}{q_{1}\left(q_{2}-1\right)}}\right)=O\left(n^{-1-\frac{1}{q_{2}-1}+\frac{q_{2}}{q_{1}\left(q_{2}-1\right)}}\right)=O\left(n^{-1-\frac{q_{1}-q_{2}}{q_{1}\left(q_{2}-1\right)}}\right),
$$

and

$$
p^{q_{1}}=O\left(n^{-1-\frac{1}{q_{2}-1}}\right) .
$$

Let $E$ be a short edge and let $E_{1}, \ldots, E_{q_{2}}$ be its covering edges. We divide the covering edges into three categories: we say that a covering edge $E_{i}$ is simple if $\left|E_{i} \cap\left(\bigcup_{j \neq i} E_{j}\right)\right|=$ 0 , we say a covering edge $E_{i}$ is generic if $\left|E_{i} \cap\left(\bigcup_{j \neq i} E_{j}\right)\right|=1$, otherwise we say this 
edge is degenerate. Let $k_{1}=\mid\left\{i: E_{i}\right.$ is simple $\} \mid$, let $k_{2}=\mid\left\{i: E_{i}\right.$ is generic $\} \mid$ and let $k_{3}=q_{2}-k_{1}-k_{2}$ be the number of degenerate edges. We are interested in counting configurations where $k_{1}<q_{2}$. We will first show that non-simple covers with $k_{3}=0$ are unlikely to occur in $H\left(n, q_{1}, q_{2}, p\right)$.

We say that a vertex is generic if it belongs to more than one generic edge and denote $m=\mid\left\{v \in V\left(H^{\prime}\right): v\right.$ is generic $\} \mid$. Since every generic vertex belongs to at least two generic edges and every generic edge contains at most one generic vertex we obtain $1 \leqslant m \leqslant \frac{k_{2}}{2}$. There are $O\left(n^{2}\right)$ many possible choices for the short edge and $O\left(n^{k_{1}}\right)$ many choices for the simple edges. Since $k_{3}=0$ every generic edge must contain a generic vertex, and thus, fixing all generic vertices determines the generic edges up to $O(1)$ many choices; thus, we have $O\left(n^{m}\right)$ many choices for the generic edges.

We require $q_{2}$ vertices for the short edge, $q_{1}-1$ vertices for every simple edge and $k_{2}\left(q_{1}-1\right)-\left(k_{2}-m\right)$ vertices for the generic edges. Hence, the expected number of such configurations is

$$
\begin{aligned}
& O\left(n^{2+k_{1}+m} p^{q_{2}+\left(k_{1}+k_{2}\right)\left(q_{1}-1\right)-\left(k_{2}-m\right)}\right)=O\left(n^{2+k_{1}+m} p^{q_{2} q_{1}-\left(k_{2}-m\right)}\right) \\
& =O\left(n^{2+k_{1}+m-\left(1+\frac{1}{q_{2}-1}\right) q_{2}+\frac{\left(k_{2}-m\right) q_{2}}{q_{1}\left(q_{2}-1\right)}}\right)=O\left(n^{2-\left(k_{2}-m\right)-\frac{q_{1} q_{2}-\left(k_{2}-m\right) q_{2}}{q_{1}\left(q_{2}-1\right)}}\right) .
\end{aligned}
$$

If $k_{2}-m \geqslant 2$, as $k_{2}-m<q_{1}$ this is clearly $o(1)$. If $k_{2}-m \leqslant 1$ then since $k_{2} \geqslant 2$ we must have $k_{2}=2$ and $m=1$ and therefore

$$
O\left(n^{2-\left(k_{2}-m\right)-\frac{q_{1} q_{2}-\left(k_{2}-m\right) q_{2}}{q_{1}\left(q_{2}-1\right)}}\right)=O\left(n^{1-\frac{q_{2}\left(q_{1}-1\right)}{q_{1}\left(q_{2}-1\right)}}\right)=O\left(n^{1-1-\frac{q_{1}-q_{2}}{q_{1}\left(q_{2}-1\right)}}\right)=o(1) .
$$

Therefore, we may assume that $k_{3}>0$. Assume first that there exists a degenerate edge $E_{1}$ along with two other covering edges $E_{2}, E_{3}$ such that $\left|E_{1} \cap E_{i}\right|=1$ for $i \in\{2,3\}$ and $\left|E_{2} \cap E_{3}\right|<2$. Then fixing $E_{1}$ determines all three edges up to $O(1)$ many choices. Thus, we have at most $O(n)$ many choices for those three edges. Since we require $q_{2}$ vertices for the short edge and $\left|E_{1} \cup E_{2} \cup E_{3}\right| \geqslant 3 q_{1}-\left|E_{1} \cap E_{2}\right|-\left|E_{1} \cap E_{3}\right|-\left|E_{2} \cap E_{3}\right|>3 q_{1}-3$, the expected number of such configurations is

$$
O\left(n^{3} p^{3 q_{1}-6+q_{2}}\right)=O\left(n^{3} p^{3\left(q_{1}-1\right)+q_{2}-3}\right)=O\left(n^{-3 \cdot \frac{q_{1}-q_{2}}{q_{1}\left(q_{2}-1\right)}} p^{q_{2}-3}\right)=o(1) .
$$

We thus deduce that every degenerate edge must either intersect some other covering edge in at least two vertices or intersect two other degenerate edges that intersect each other in at least two vertices. Therefore, $k_{3} \geqslant 2$, and by Lemma 36 we have at most $O(1)$ many choices for the degenerate edges.

We now divide the generic edges into two categories: We say a generic edge is weaklygeneric if it is a generic edge that lies in the same connected component of $H^{\prime} \backslash\{E\}$ as a degenerate edge; otherwise, we say it is strongly-generic. We denote $k_{2}^{\prime}=\mid\{i$ : $E_{i}$ is strongly-generic $\} \mid$. We say that a vertex is strongly-generic if it belongs to more than one strongly-generic edge, and denote $m^{\prime}=\mid\left\{v \in V\left(H^{\prime}\right): v\right.$ is strongly-generic $\} \mid$. Since every strongly-generic vertex belongs to at least two strongly-generic edges and every strongly-generic edge contains at most one strongly-generic vertex we obtain $0 \leqslant$ $m^{\prime} \leqslant \frac{k_{2}^{\prime}}{2} \leqslant \frac{k_{2}}{2}$. Again, we note that fixing the strongly-generic vertices determines the 
strongly-generic edges up to $O(1)$ many choices. We also note that fixing $E$ determines the degenerate and weakly-generic edges up to $O(1)$ many choices. Since, by Lemma 39 , we require at least $2 q_{1}\left(1-\frac{1}{k_{3}}\right)$ vertices for the degenerate edges, at least $k_{2}\left(q_{1}-1\right)$ additional vertices for the generic edges and $k_{1} q_{1}$ vertices for the simple edges, we get that the expected number of such configurations is (since $k_{1}+k_{2}+k_{3}=q_{2}, k_{3} \geqslant 2$ and $\left.m^{\prime} \leqslant \frac{k_{2}}{2}\right)$

$$
\begin{aligned}
& O\left(n^{2+k_{1}+m^{\prime}} p^{k_{1} q_{1}+k_{2}\left(q_{1}-1\right)+2 q_{1}\left(1-\frac{1}{k_{3}}\right)}\right) \\
& =O\left(n^{2+k_{1}+m^{\prime}-k_{1}\left(1+\frac{1}{q_{2}-1}\right)-k_{2}\left(1+\frac{q_{1}-q_{2}}{q_{1}\left(q_{2}-1\right)}\right)} p^{2 q_{1}\left(1-\frac{1}{k_{3}}\right)}\right) \\
& =O\left(n^{2-k_{1} \frac{1}{q_{2}-1}+m^{\prime}-k_{2}-k_{2} \frac{q_{1}-q_{2}}{q_{1}\left(q_{2}-1\right)}} p^{2 q_{1}\left(1-\frac{1}{k_{3}}\right)}\right),
\end{aligned}
$$

since $m^{\prime}-k_{2} \leqslant-\frac{k_{2}}{2}$ and $p^{2 q_{1}\left(1-\frac{1}{k_{3}}\right)}=O\left(n^{-2-\frac{2}{q_{2}-1}+\frac{2 q_{2}}{k_{3}\left(q_{2}-1\right)}}\right)$,

$$
\begin{aligned}
& =O\left(n^{2-\frac{k_{1}}{q_{2}-1}-\frac{k_{2}}{2}-k_{2} \frac{q_{1}-q_{2}}{q_{1}\left(q_{2}-1\right)}-2-\frac{2}{q_{2}-1}+\frac{2 q_{2}}{k_{3}\left(q_{2}-1\right)}}\right) \\
& =O\left(n^{-k_{2} \frac{q_{1}-q_{2}}{q_{1}\left(q_{2}-1\right)}-\frac{\left(2+k_{1}+\frac{k_{2}}{2}\left(q_{2}-1\right)\right) k_{3}-2 q_{2}}{k_{3}\left(q_{2}-1\right)}}\right) \\
& =O\left(n^{-k_{2} \frac{q_{1}-q_{2}}{q_{1}\left(q_{2}-1\right)}-\frac{2 k_{3}+k_{1} k_{3}+k_{2} k_{3}-2 q_{2}}{k_{3}\left(q_{2}-1\right)}}\right)=o(1) .
\end{aligned}
$$

Finally, we conclude by Markov's inequality,

$$
\mathbb{P}[\# \text { short edges with non-simple covers }>0]=\sum_{k_{1}<q_{2}} \sum_{k_{2}<q_{2}-k_{1}} o(1)=o(1) .
$$

Lemma 43. Let $H \subset H\left(n, q_{1}, q_{2}\right)$ be a simple path of length $\ell=O(\log n)$. Define $Y=$ $\mid\left\{T \subset H\left(n, q_{1}, q_{2}\right): H \cup T\right.$ is a simple path of length $\left.\ell+1\right\} \mid$. Then for every $c_{r}>0$, there exists $c>0$ such that for $p \leqslant c \cdot n^{-\frac{q_{2}}{q_{1}\left(q_{2}-1\right)}}$ we have

$$
\mathbb{E}\left[Y \mid V(H) \subset[n]_{p}\right]<c_{r} .
$$

Proof. Without loss of generality we may assume that the short edge in $T$ interesects $H$ only at $E_{\ell, q_{2}}$. We denote $T=\left\{E=E_{\ell+1}, E_{\ell+1,2}, \ldots, E_{\ell+1, q_{2}}\right\}$. Since $E$ intersects $H$ in a single vertex we have $O(n)$ many choices for it. By fixing $E$ we limit the number of choices for each covering edge to $O(n)$, giving us a total of $O\left(n^{q_{2}}\right)$ many choices for $T$ in $H\left(n, q_{1}, q_{2}\right)$.

Since $T$ requires $q_{2}-1+\left(q_{2}-1\right)\left(q_{1}-1\right)=\left(q_{2}-1\right) q_{1}$ new vertices, we obtain

$$
\mathbb{E}\left[Y \mid V(H) \subset[n]_{p}\right]=O\left(n^{q_{2}} p^{\left(q_{2}-1\right) q_{1}}\right)=O\left(n^{q_{2}-q_{2}} c^{\left(q_{2}-1\right) q_{1}}\right)=O\left(c^{\left(q_{2}-1\right) q_{1}}\right) .
$$

Corollary 44. Let $\ell=O(\log n)$, and let $Y$ be the random variable counting simple paths of length $\ell$ in $H\left(n, q_{1}, q_{2}, p\right)$. Then,

$$
\mathbb{E} Y=O\left(c_{r}^{\ell} n^{1-\frac{1}{q_{2}-1}}\right)
$$


Proof. We may construct a simple path of length $\ell$ by first choosing a single long edge, and then choosing $\ell$ extensions.

Since two integers belong to only a constant number of $q_{1}$-term arithmetic progressions, we obtain that the number of choices for the initial long edge is $O\left(n^{2}\right)$. Since we require $q_{1}$ vertices for the long edge, we obtain that the expected number of long edges is $O\left(n^{2} p^{q_{1}}\right)=$ $O\left(n^{1-\frac{1}{q_{2}-1}}\right)$.

By the previous lemma, we may extend the path step by step, each time adding a factor of $c_{r}$ to the expectation, and the corollary immediately follows.

We are now ready to prove the probabilistic lemma, thus completing the proof of Theorem 26.

Proof of The probabilistic lemma. Let $H=H\left(n, q_{1}, q_{2}, p\right)$, let $B$ be a sufficiently large constant and set $\ell^{\prime}=B \log n$. The proof of this lemma can be summarized as follows:

First, we recall that, by Lemma 42, a.a.s no non-simply covered short edges exist, and show that all simple paths terminate at lengths smaller than $\ell^{\prime}$, using Corollary 44 . We then apply a first-moment argument to several random variables, showing that every small 2-blocking hypergraph contains some sub-hypergraph with $o(1)$ many expected copies in $H$; thus, the probability that the 2-blocking hypergraph appears in our random hypergraph is $o(1)$.

Specifically, we will show that the existence of a 2-blocking hypergraph implies either the existence of additional vertices such that the expected number of choices for them is $o\left(n^{-1+\frac{1}{q_{2}-1}}\right)$, or the existence of a simple path that obeys an additional constraint that causes us to lose a degree of freedom in the path construction. Since, by Corollary 44, we have only an expected $O\left(n^{1-\frac{1}{q_{2}-1}}\right)$ many choices for a simple path, we obtain that replacing a factor of $n$ with a factor of $O\left(\log ^{k} n\right)$ causes the expectation to tend to zero as $n$ grows.

As seen in the proof for Corollary 44, we may construct a path by selecting a long edge and then iteratively extending the path; therefore, in some cases, we refer to a single long edge as a simple path of length zero, allowing us to treat a single block as an extension to an existing path.

We will also sometimes assume that the edges of a simple path are ordered $E_{1,1}, E_{1}$, $E_{1,2}, \ldots, E_{1, q_{2}}, E_{2}, \ldots, E_{\ell, q_{2}}$. In such an ordering, if there are no other constraints on the path, we have $O\left(n^{2}\right)$ many choices for the first edge and $O(n)$ many choices for every other edge. For convenience, we say a vertex $v \in V(P)$ precedes another vertex $u \in V(P)$ if $v$ belongs to an edge that precedes all edges that contain $u$.

Let $U$ be the random variable counting short edges with non-simple covers. By Lemma 42 we have

$$
\mathbb{P}(U>0)=o(1) .
$$

Let $W$ be the random variable counting simple paths of length $\ell^{\prime}$. By Corollary 44 and Markov's inequality we obtain

$$
\mathbb{P}(W>0) \leqslant \mathbb{E} W=O\left(n^{1-\frac{1}{q_{2}-1}} c_{r}^{B \log n}\right)=o(1) .
$$


Let $X$ be the random variable counting spoiled simple paths of length $\ell<\ell^{\prime}$. Let $P$ be a simple path and let $E$ be a spoiling edge for it. By Corollary 44, there are at most $O\left(n^{1-\frac{1}{q_{2}-1}}\right)$ many choices for the path up to the final block. For the final block we have $O(n)$ many choices for the short edge, and for all the long edges except $E_{\ell, q_{2}}$. Since $\left|E \cap\left(V(P) \backslash E_{\ell, q_{2}}\right)\right| \geqslant 2$ we have at most $O\left(\log ^{2} n\right)$ many choices for $E$, and therefore $O\left(\log ^{2} n\right)$ many choices for $E_{\ell, q_{2}}$. Since we require $q_{2}-1$ vertices for the short edge, along with $\left(q_{2}-1\right)\left(q_{1}-1\right)$ vertices for the long edges we conclude that

$$
\mathbb{E} X=\sum_{\ell<\ell^{\prime}} O\left(n^{1-\frac{1}{q_{2}-1}} n^{q_{2}-1} p^{q_{1}\left(q_{2}-1\right)} \log ^{2} n\right)=O\left(n^{q_{2}-q_{2}-\frac{1}{q_{2}-1}} \log ^{2} n\right)=o(1),
$$

and therefore by Markov's inequality,

$$
\mathbb{P}(X>0)=o(1)
$$

Let $Y$ be the random variable counting simple paths of length $\ell<\ell^{\prime}$ with a saw. We denote the path by $P$. Since the saw edges are not entirely contained in $P$, each one must contain a vertex $v \notin V(P)$. Let $S_{2}, \ldots, S_{q_{1}}$ be the saw edges. For each $k \in\left\{2, \ldots, q_{1}\right\}$, let $\left\{s_{k}\right\}=\left(V(P) \cap S_{k}\right) \backslash E_{1,1}$ (there is only one such vertex, since $\left|S_{k} \cap V(P)\right|=2$ ), and let $S_{k}^{\prime}$ be the first edge in $P$ that contains $s_{k}$. We split into several cases:

Case 1. There exist two edges $S_{i}, S_{j}$ such that $\left|S_{i} \cap S_{j}\right| \geqslant 2$.

By Lemma 36, we obtain that fixing $E_{1,1}$ determines $S_{i}$ and $S_{j}$ up to $O(1)$ many choices. Thus, if we iteratively extend a path from $E_{1,1}$ we obtain that when we select $S_{i}^{\prime}$ it must intersect one (or both) of $S_{i}, S_{j}$, giving us only $O(1)$ many choices for it, and thus the expected number of such paths with $S_{i}$ and $S_{j}$ in $H$ is $O\left(n^{-\frac{1}{q_{2}-1}} \log n\right)=o(1)$. Therefore, the probability that such saw edges exist is $o(1)$ and we may assume all saw edges $S_{i}$ and $S_{j}$ intersect each other in at most one vertex.

Case 2a. There exist $i, j$ such that $\left(S_{i} \cap S_{j}\right) \backslash V(P) \neq \emptyset$ and $S_{i}^{\prime} \neq S_{j}^{\prime}$.

Without loss of generality we assume $S_{i}^{\prime}$ follows $S_{j}^{\prime}$ in the edge ordering of $P$. If we fix the subpath up to (but not including) $S_{i}^{\prime}$, then we have at most $O(\log n)$ many choices for $S_{j}$ since it intersects both $E_{1,1}$ and $S_{j}^{\prime}$ which have already been chosen; this implies that there are further $O(1)$ many choices for $S_{i}$, as it must intersect both $S_{j}$ and $E_{1,1}$. Therefore, there are further $O(1)$ many choices for $S_{i}^{\prime}$ as it must intersect both $S_{i}$ and either a short edge or the final long edge of the previous block. We now have $O(n)$ many choices for each remaining edge in the block that contains $S_{i}^{\prime}$.

We obtain that for the final block, we have $O(n)$ many choices for each edge except for $S_{i}^{\prime}$ for which we have only $O(\log n)$ many choices. Hence the expected number of such configurations is

$$
\sum_{\ell<\ell^{\prime}} O\left(n^{1-\frac{1}{q_{2}-1}} n^{q_{2}-1} p^{q_{1}\left(q_{2}-1\right)} \log n\right)=O\left(n^{q_{2}-q_{2}-\frac{1}{q_{2}-1}} \log ^{2} n\right)=o(1) .
$$

Case 2b. There exist $i, j$ such that $\left(S_{i} \cap S_{j}\right) \backslash V(P) \neq \emptyset$ and $S_{i}^{\prime}=S_{j}^{\prime}=S^{\prime} \neq E_{1}$. 
By fixing $E_{1,1}$ and $\{v\}=S_{i} \cap S_{j}$ we determine $S_{i}$ and $S_{j}$ up to $O(1)$ many choices. Since $v \notin V(P)$, we also determine $S^{\prime}$ up to $O(1)$ many choices as it must intersect $S_{i} \cup S_{j}$ in two vertices other than $v$. If we now extend a path from $E_{1,1}$ towards $S^{\prime}$, we obtain that we have only $O(1)$ many choices for the edge connecting the path to $S^{\prime}$ (whether it is a short edge, or the final long edge in a block). Note that this connecting edge cannot be $E_{1,1}$ by our assumption that $S^{\prime} \neq E_{1}$. Thus, the number of choices for both $S^{\prime}$ and the previous edge is $O(n)$ - the number of choices for $v$.

If we now add the remaining edges in the final block, we obtain that the expected number of such paths is

$$
O\left(n^{-\frac{1}{q_{2}-1}} \log n\right)=o(1) .
$$

Case 2c. There exist $i, j$ such that $\left(S_{i} \cap S_{j}\right) \backslash V(P) \neq \emptyset$ and $S_{i}^{\prime}=S_{j}^{\prime}=S^{\prime}=E_{1}$.

From the previous cases we may assume that all saw edges intersect each other in at most one vertex, and any saw edge that intersects another saw edge outside of $V(P)$ must intersect $V(P) \backslash E_{1,1}$ in one vertex that lies on $E_{1}$.

Let $A=\bigcup_{i=2}^{q_{1}}\left(S_{i} \backslash V(P)\right)$ and for each $v \in A$ define $S(v)=\left\{i: v \in S_{i}\right\}$. Suppose that $|S(v)| \geqslant 2$ for some $v \in A$. Then,

$$
\left|\bigcup_{i \in S(v)}\left(S_{i} \cap\left(E_{1} \backslash E_{1,1}\right)\right)\right|=|S(v)|
$$

since no two saw edges share more than one vertex and any saw edge that intersects another has a vertex in $E_{1} \backslash E_{1,1}$. Therefore, $|S(v)| \leqslant\left|E_{1} \backslash E_{1,1}\right|=q_{2}-1$ for all $v \in A$. Since,

$$
\left(q_{2}-2\right)\left(q_{1}-1\right)=\sum_{i=2}^{q_{1}}\left|S_{i} \backslash V(P)\right|=\sum_{v \in A}|S(v)| \leqslant|A|\left(q_{2}-1\right),
$$

we deduce that we require $|A| \geqslant \frac{\left(q_{2}-2\right)\left(q_{1}-1\right)}{q_{2}-1}=q_{1}-1-\frac{q_{1}-1}{q_{2}-1}$ additional vertices for the saw edges.

If we assume $P$ is fixed, we have at most $O(\log n)$ many choices for each saw edge. Thus, the expected number of choices for the additional vertices is

$$
\begin{aligned}
O\left(\log ^{q_{1}-1} n \cdot p^{q_{1}-1-\frac{q_{1}-1}{q_{2}-1}}\right) & =O\left(\log ^{q_{1}-1} n \cdot n^{-1-\frac{1}{q_{2}-1}+\frac{q_{2}}{q_{1}\left(q_{2}-1\right)}+\frac{q_{1} q_{2}}{q_{1}\left(q_{2}-1\right)^{2}}-\frac{q_{2}}{q_{1}\left(q_{2}-1\right)^{2}}}\right) \\
& =O\left(\log ^{q_{1}-1} n \cdot n^{-1-\frac{q_{1}-q_{2}}{q_{1}\left(q_{2}-1\right)}+\frac{1}{q_{2}-1}+\frac{q_{1}-q_{2}}{q_{1}\left(q_{2}-1\right)^{2}}}\right) \\
& =O\left(\log ^{q_{1}-1} n \cdot n^{-1+\frac{1}{q_{2}-1}-\frac{\left(q_{1}-q_{2}\right)\left(q_{2}-2\right)}{q_{1}\left(q_{2}-1\right)^{2}}}\right) .
\end{aligned}
$$

Thus by Corollary 44, the expected number of choices for the path and the saw edges is

$$
O\left(\log ^{q_{1}} n \cdot n^{-\frac{\left(q_{1}-q_{2}\right)\left(q_{2}-2\right)}{q_{2}\left(q_{2}-1\right)^{2}}}\right)=o(1) .
$$

Case 3. $\left(S_{i} \cap S_{j}\right) \backslash V(P)=\emptyset$ for all $i \neq j \in\left\{2, \ldots, q_{1}\right\}$.

Since each saw edge contains at least one vertex not in $V(P)$, there are at least $q_{1}-1$ additional vertices introduced by the saw edges. We have $O(\log n)$ many choices for each 
saw edge as they must intersect $V(P)$ in two vertices each, one of which lies on $E_{1,1}$. Thus, the expected number of paths with saws such as above is

$$
\begin{aligned}
\sum_{\ell<\ell^{\prime}} O\left(n^{1-\frac{1}{q_{2}-1}}(\log n \cdot p)^{q_{1}-1}\right) & =O\left(\log ^{q_{1}} n \cdot n^{1-\frac{1}{q_{2}-1}-\frac{q_{2}}{q_{2}-1}+\frac{q_{2}}{q_{1}\left(q_{2}-1\right)}}\right) \\
& =O\left(\log ^{q_{1}} n \cdot n^{-\frac{2 q_{1}-q_{2}}{q_{1}\left(q_{2}-1\right)}}\right)=o(1) .
\end{aligned}
$$

Thus, by Markov's inequality,

$$
\mathbb{P}(Y>0)=o(1)
$$

Finally, let $Z$ be the random variable counting simple paths of length $\ell<\ell^{\prime}$ with a spoiled extension. Once more, we divide the argument into several cases. First, let $Z_{1}$ be the random variable counting simple paths with spoiled extensions such that no long edge in the extension intersects the path $P$ in more than one vertex.

We denote the number of long edges in the extension that intersect $V(P)$ by $k$. We have an expected $O\left(n^{1-\frac{1}{q_{2}-1}}\right)$ many choices for $P, O(n)$ many choices for the short edge and each of the $q_{2}-1-k$ long edges that are disjoint from $V(P)$, and $O(\log n)$ many choices for each of the $k$ long edges that intersect $P$. We also require $k\left(q_{1}-1\right)+\left(q_{2}-1-k\right) q_{1}$ new vertices. Thus, the expected number of such configurations is

$$
\begin{aligned}
\mathbb{E} Z_{1} & =\sum_{\ell<\ell^{\prime}} \sum_{k=1}^{q_{2}} O\left(n^{1-\frac{1}{q_{2}-1}+q_{2}-k} p^{\left(q_{2}-1-k\right) q_{1}+k\left(q_{1}-1\right)} \log ^{k} n\right) \\
& =\sum_{k=1}^{q_{2}} O\left(n^{1-\frac{1}{q_{2}-1}+q_{2}-k} p^{\left(q_{2}-1\right) q_{1}-k} \log ^{q_{2}+1} n\right) \\
& =\sum_{k=1}^{q_{2}} O\left(n^{1-\frac{1}{q_{2}-1}-k\left(1-\frac{q_{2}}{q_{1}\left(q_{2}-1\right)}\right)} \log ^{q_{2}+1} n\right) \\
& =O\left(n^{-\frac{q_{1}-q_{2}}{q_{1}\left(q_{2}-1\right)}} \log ^{q_{2}+1} n\right)=o(1) .
\end{aligned}
$$

Next, let $Z_{2}$ be the random variable counting paths with spoiled extensions that contain exactly one long edge $L$ that intersects the path $P$ in at least two vertices. Fixing $P$, we have only $O\left(\log ^{2} n\right)$ many choices for $L$ and $O(1)$ further choices for $E_{\ell+1}$, as it must intersect both $L$ and $E_{\ell, q_{2}}$. Once again, we sum over $k$, the number of long edges in the extension that intersect $V(P)$ in exactly one vertex. We have $O\left(n^{q_{2}-2-k}\right)$ choices for all edges of the extension, and we require at least $\left(q_{2}-2\right) q_{1}-k+1$ new vertices. Thus, since $k \leqslant q_{2}-2$ and

$$
p^{q_{1}\left(q_{2}-2\right)}=O\left(n^{-\frac{q_{2}\left(q_{2}-2\right)}{q_{2}-1}}\right)=O\left(n^{-q_{2}+1+\frac{1}{q_{2}-1}}\right),
$$

the expected number of such configurations is

$$
\begin{aligned}
\mathbb{E} Z_{2} & =\sum_{\ell<\ell^{\prime}} \sum_{k=0}^{q_{2}-2} O\left(n^{q_{2}-k-1-\frac{1}{q_{2}-1}} p^{\left(q_{2}-2\right) q_{1}-k+1} \log ^{2} n\right) \\
& =\sum_{k=0}^{q_{2}-2} O\left(n^{-k} p^{1-k} \log ^{3} n\right)=O\left(\log ^{3} n \cdot p\right)=o(1) .
\end{aligned}
$$


Finally, let $Z_{3}$ be the random variable counting paths with spoiled extensions such that there exist distinct $i, j \in\left[q_{2}\right]$ such that $\left|V(P) \cap E_{\ell+1, z}\right| \geqslant 2$ for $z \in\{i, j\}$. We denote $L_{z}=E_{\ell+1, z}$.

Since $E_{\ell+1}$ must have a simple cover we obtain that $L_{z} \cap E_{\ell, q_{2}}=\emptyset$ for $z \in\{i, j\}$. Therefore, if we fix the path $P$ up to (but not including) $E_{\ell, q_{2}}$, we have at most $O\left(\log ^{2} n\right.$ ) many choices for each of $L_{i}$ and $L_{j}$, since both edges intersect $V(P)$ in at least two vertices. Therefore, we have at most $O\left(\log ^{4} n\right)$ many choices for $E_{\ell+1}$ as it must intersect both $L_{i}$ and $L_{j}$.

Finally, we obtain that we have $O\left(\log ^{4} n\right)$ many choices for $E_{\ell, q_{2}}$ as it must intersect both $E_{\ell+1}$ and $E_{\ell}$. Since we have an expected $O\left(n^{1-\frac{1}{q_{2}-2}}\right)$ many choices for the path up to the final block, and we have $O\left(n^{q_{2}-1} \log ^{4} n\right)$ many choices for the final block (but still require $q_{1}\left(q_{2}-1\right)$ vertices $)$, the expected number of choices for $P$ is

$$
\sum_{\ell<\ell^{\prime}} O\left(n^{-\frac{1}{q_{2}-1}} \log ^{4} n\right)=o(1)
$$

hence

$$
\mathbb{P}\left(Z_{3}>0\right)=o(1)
$$

and therefore

$$
\mathbb{P}(Z>0) \leqslant \mathbb{P}\left(Z_{1}+Z_{2}+Z_{3}>0\right)=o(1)
$$

Finally,

$$
\mathbb{P}(U=W=X=Y=Z=0) \rightarrow 1 \text { as } n \rightarrow \infty
$$

completing the proof.

\section{Acknowledgements}

I would like to thank my advisor Wojciech Samotij for his introduction to this problem and for his guidance towards its solution. I would also like to express my deepest appreciation for his kindness, patience and meticulousness during the research and writing process.

\section{References}

[1] Elad Aigner-Horev and Yury Person, An asymmetric random Rado theorem: 1statement, arXiv: 1906.05614.

[2] Noga Alon and Joel H. Spencer, The probabilistic method, fourth ed., Wiley Series in Discrete Mathematics and Optimization, John Wiley \& Sons, Inc., Hoboken, NJ, 2016. MR 3524748

[3] József Balogh, Robert Morris, and Wojciech Samotij, Independent sets in hypergraphs, J. Amer. Math. Soc. 28 (2015), no. 3, 669-709. MR 3327533

[4] - The method of hypergraph containers, Proceedings of the International Congress of Mathematicians-Rio de Janeiro 2018. Vol. 3, 2018, pp. 3045-3078. 
[5] Péter Frankl and Vojtěch Rödl, Large triangle-free subgraphs in graphs without $K_{4}$, Graphs Combin. 2 (1986), no. 2, 135-144. MR 932121

[6] Ehud Friedgut, Hiệp Hàn, Yury Person, and Mathias Schacht, A sharp threshold for van der Waerden's theorem in random subsets, Discrete Anal. (2016), Paper No. 7, 20. MR 3533306

[7] Luca Gugelmann, Rajko Nenadov, Yury Person, Nemanja Škorić, Angelika Steger, and Henning Thomas, Symmetric and asymmetric Ramsey properties in random hypergraphs, Forum Math. Sigma 5 (2017), e28, 47. MR 3725732

[8] Robert Hancock and Andrew Treglown, An asymmetric random Rado theorem for single equations: the 0-statement, arXiv:2004.14076v1.

[9] T. E. Harris, A lower bound for the critical probability in a certain percolation process, Proc. Cambridge Philos. Soc. 56 (1960), 13-20. MR 0115221

[10] Svante Janson, Poisson approximation for large deviations, Random Structures Algorithms 1 (1990), no. 2, 221-229. MR 1138428

[11] Yoshiharu Kohayakawa and Bernd Kreuter, Threshold functions for asymmetric Ramsey properties involving cycles, Random Structures Algorithms 11 (1997), no. 3, 245-276. MR 1609513

[12] Anita Liebenau, Letícia Mattos, Walner Mendonça, and Jozef Skokan, Asymmetric ramsey properties of random graphs for cliques and cycles, 2020.

[13] Martin Marciniszyn, Jozef Skokan, Reto Spöhel, and Angelika Steger, Asymmetric Ramsey properties of random graphs involving cliques, Random Structures Algorithms 34 (2009), no. 4, 419-453. MR 2531778

[14] Frank Mousset, Rajko Nenadov, and Wojciech Samotij, Towards the KohayakawaKreuter conjecture on asymmetric Ramsey properties, Combinatorics, Probability and Computing 29 (2020), no. 6, 943-955.

[15] Rajko Nenadov and Angelika Steger, A short proof of the random Ramsey theorem, Combin. Probab. Comput. 25 (2016), no. 1, 130-144. MR 3438289

[16] Richard Rado, Studien zur Kombinatorik, Mathematische Zeitschrift (1933), 424470.

[17] Vojtěch Rödl and Andrzej Ruciński, Lower bounds on probability thresholds for Ramsey properties, Combinatorics, Paul Erdős is eighty, Vol. 1, Bolyai Soc. Math. Stud., János Bolyai Math. Soc., Budapest, 1993, pp. 317-346. MR 1249720

[18] _ Random graphs with monochromatic triangles in every edge coloring, Random Structures Algorithms 5 (1994), no. 2, 253-270. MR 1262978

[19] - Threshold functions for Ramsey properties, J. Amer. Math. Soc. 8 (1995), no. 4, 917-942. MR 1276825

[20] _ Rado partition theorem for random subsets of integers., Proceedings of the London Mathematical Society 74 (1997), no. 3, N.PAG.

[21] David Saxton and Andrew Thomason, Hypergraph containers, Invent. Math. 201 (2015), no. 3, 925-992. MR 3385638 
[22] Bartel Leendert Van der Waerden, Beweis einer Baudetschen Vermutung, Nieuw. Arch. Wisk. (1927).

[23] Panayiotis Varnavides, On certain sets of positive density, J. London Math. Soc. 34 (1959), 358-360. MR 106865 